\title{
Determination of the internal structure of neutron stars from gravitational wave spectra
}

\author{
L.K. Tsui, P.T. Leung*, and J. Wu \\ Physics Department and Institute of Theoretical Physics, \\ The Chinese University of Hong Kong, Shatin, Hong Kong SAR, China.
}

(Dated: July 6, 2018)

\begin{abstract}
In this paper the internal structure of a neutron star is shown to be inferrable from its gravitational-wave spectrum. Iteratively applying the inverse scheme of the scaled coordinate logarithmic perturbation method for neutron stars proposed by Tsui and Leung [Astrophys. J. 631, 495 (2005)], we are able to determine the mass, the radius and the mass distribution of a star from its quasi-normal mode frequencies of stellar pulsation. In addition, accurate equation of state of nuclear matter can be obtained from such inversion scheme. Explicit formulas for the case of axial $w$-mode oscillation are derived here and numerical results for neutron stars characterized by different equations of state are shown.
\end{abstract}

PACS numbers: 04.40.Dg, 04.30.Db, 97.60.Jd, 95.30.Sf

\section{INTRODUCTION}

As remnants of supernova explosions, neutron stars comprised of matters with subnuclear and supranuclear densities have long been a major subject of intense interest for researchers in astrophysics, nuclear physics and particle physics since the pioneering studies of Oppenheimer and Volkoff [1] and Tolman 2]. Their internal structure is likely to provide a direct test for theories of nuclear matter, quark matter and high energy physics (see e.g. 3, 4, 5] and references therein). For example, through measurement of the Eddington flux and the redshift in the X-ray burst, the mass and the radius of the neutron star EXO 0748-676 were simultaneous determined, and relevant data were used to rule out soft equations of state (EOS) for nuclear matter [6, 7]. Likewise, a few astronomical observations revealing evidences for the existence of the strange quark star (SQS), a variant of the neutron star (see e.g. [8, 9, 10]), to some extent resuscitate the theory of quark matter [1, 12, 13, 14].

On the other hand, the huge gravitational field accompanying a dense neutron star also attracts the attention of relativists. In particular, undulating neutron stars are expected to be promising sources of gravitational waves. For instance, when gravitational-wave detectors of various designs are in full swing in one or two decades (see e.g. [15, 16] and references therein), the frequency of detection of gravitational waves emitted in the mergers of binary neutron stars could be as high as several hundreds per year [15, 17, 18]. Besides, gravitational waves emitted during asymmetric stellar core collapses leading to the formation of neutron stars could also be detectable [19, 20]. It is then likely that neutron stars could be surveyed in the gravitational wave channel. More importantly, gravitational waves can easily penetrate the core of a neutron star without being absorbed, and might carry useful information about the deep interior of the star. As the behavior of nuclear matter at high densities is not yet clearly known, the internal structure of neutron stars is masked and often gives rise to many debatable issues. Hence, a lot of in-depth studies have been sparked off by the possibility of unveiling the internal structure of neutron stars from their gravitational-wave signals. Detailed examination of such signals, which has been coined "gravitational-wave asteroseismology", were carried out by various groups of researchers (see e.g. 21, 22, 23, 24, 25, 26, 27, 28, 29]) to study effects of different physical processes, including superfluidity, occurrence of quark matter, and phase transitions.

Gravitational waves emitted from a neutron star are commonly analyzed in terms of quasi-normal modes (QNMs) characterized by complex eigenfrequencies $\omega=\omega_{r}-i \omega_{i}$, with $\omega_{r}$ and $\omega_{i}$ measuring respectively the rates of pulsation and damping [30, 31]. To infer the physical parameters of a neutron star from its QNM frequencies, Andersson and Kokkotas made use of the universal behavior in the QNMs of the fundamental fluid $f$-mode and the first polar $w$-mode to estimate the radius and the mass of the star 22]. Besides, the most probable EOS among all the known models of stellar matters could also be identified from the frequency of the leading $p$-mode (or axial $w$-mode) oscillation 22, 23].

In a series of recent papers [32, 33], we have studied the physical origin of the universality in the pulsation frequencies of the $f$-mode and $w$-mode stellar pulsations discovered in Refs. [22] and [23], and developed a scaled-coordinate logarithmic perturbative theory (SCLPT) for neutron stars to locate axial $w$-mode oscillations. The main objective of the current paper is then to work out a feasible scheme to infer the internal structure of a neutron star from its

\footnotetext{
* Email: ptleung@phy.cuhk.edu.hk
} 
gravitational wave spectrum. Assuming that the frequencies and the damping rates of a few $w$-mode oscillations of a neutron star can be identified from gravitational-wave observation, we iteratively invert the SCLPT scheme to determine the mass $M$, the radius $R$, the mass density $\rho(r)$ at radius $r$, and the mass distribution function $m(r)$ (i.e. the total mass inside radius $r$ ) of the star. In turn, accurate equation of state of nuclear matter can also be obtained numerically from the mass distribution function. While the idea underlying the inversion scheme reported here is completely generic, we will consider and use the spectrum of axial $w$-mode oscillations to illustrate our method. As a remark, we note here that Lindblom has proposed a scheme to determine, up to certain accuracy, EOS of the nuclear matter of a neutron star from its mass and radius [34]. However, the EOS considered in Lindblom's scheme is limited to barotropic type. In contrast, our method directly exploit the frequencies of several QNMs to determine the radius, the mass and other useful parameters of the star. Moreover, the EOS obtained from our inversion scheme is free from constraints. We will show in the following discussion that even the exotic EOS of quark matter can be inferred from the gravitational wave signals of a SQS.

The organization of our paper is as follows. In Sect. II, we review the universality existing in the QNM frequencies (including the real and the imaginary parts) of neutron stars described by different EOS and discuss how such behavior can be explained by the Tolman VII model (TVIIM) of stars [2]. In Sect. III we approximate the mass distribution of a realistic neutron star by that of TVIIM plus some small correction terms to be determined from the inversion scheme. Section IV outlines the spirit of the inversion scheme while explicit formulas for the scheme are derived in Sect. V. In Sect. VI we apply our method to invert the EOSs of several realistic stars and numerical results are shown there. Section VII studies the feasibility of our method and then we conclude our paper in Sect. VIII. The present paper is the expanded version of a recent Letter [35]. Unless otherwise stated, geometric units are used in this paper.

\section{UNIVERSALITY AND TVIIM}

First of all, we review the universality in QNMs of gravitational waves and state its physical origin, thus introducing the starting point of our inversion scheme - the TVIIM. It has recently been observed that the QNM frequencies of the leading $w$-mode and $f$-mode oscillations of non-rotating neutron stars approximately satisfy the following scaling law [22, 23]:

$$
M \omega=a\left(\frac{M}{R}\right)^{2}+b\left(\frac{M}{R}\right)+c .
$$

Here $a, b$, and $c$ are complex constants determined from curve fitting. In Fig. 四 we plot $M \omega_{r}$ and $M \omega_{i}$ against the compactness $\mathcal{C} \equiv M / R$ for the leading axial $w$-mode of non-rotating neutron stars characterized by different EOSs, including models A and C proposed by Pandharipande [36, 37], models AU and UT proposed by Wiringa, Fiks and Fabrocini [38], models APR1 and APR2 proposed by Akmal, Pandharipande and Ravenhall [4]. One can easily see that both $M \omega_{r}$ and $M \omega_{i}$ are, in agreement with (2.1), well approximated by quadratic function of $\mathcal{C}$.

The universality observed in the spectra of realistic neutron stars comes as a surprise because, as shown in Fig. 2 the pressure $P$ at a given density $\rho$ varies greatly for the EOSs considered there. To understand the physical origin underlying the universality, we showed in Ref. [32] that the scaled complex eigenfrequencies $M \omega$ of axial $w$-mode, polar $w$-mode and $f$-mode oscillations, to a good approximation, depend only on the compactness $M / R$. In addition, we also discovered that the TVIIM, which was first proposed by Tolman in 1939 [2] and has a mass distribution function given by:

$$
m_{c}(r)=M\left[\frac{5}{2}\left(\frac{r}{R}\right)^{3}-\frac{3}{2}\left(\frac{r}{R}\right)^{5}\right],
$$

can mimic the mass distribution of most neutron stars. As clearly shown in Fig. 3 where the normalized mass function $m(r) / M$ is plotted against the scaled radius $r / R$ for stars with a compactness $M / R=0.28$ and described by different EOS as mentioned above, the mass distribution function $m_{c}(r)$ is able to approximate the mass distribution inside neutron stars with different EOSs. Moreover, the scaled frequency $M \omega$ of QNMs of TVIIM is close to those of realistic neutron stars (see Fig. (1) and display similar scaling behavior mentioned above. It is obvious that TVIIM does provide a good approximation to stars with varying EOSs, and the universality shown in Fig. 1 can be captured by the best quadratic fit to the QNMs of TVIIM, with $a=-4.4-6.3 \mathrm{i}, b=3.1+1.9 \mathrm{i}$, and $c=-0.072+0.098 \mathrm{i}$. A distinct feature of TVIIM is that the scaled complex eigenfrequencies $M \omega$ of its QNMs depend only on the compactness of the star 32]. Hence, the observed universality in the QNMs ( $w$-mode and $f$-mode) is expected to be closely related to TVIIM and was indeed verified using SCLPT [33].

The density distribution of TVIIM, given by

$$
\rho(r)=\rho_{0}\left[1-\left(\frac{r}{R}\right)^{2}\right],
$$


with the central density $\rho_{0}=15 M /\left(8 \pi R^{3}\right)$, is so simple that the metric coefficients $e^{\nu(r)}$ and $e^{\lambda(r)}$, defined by the line element $\mathrm{d} s$ :

$$
\mathrm{d} s^{2}=-e^{\nu(r)} \mathrm{d} t^{2}+e^{\lambda(r)} \mathrm{d} r^{2}+r^{2}\left(\mathrm{~d} \theta^{2}+\sin ^{2} \theta \mathrm{d} \varphi^{2}\right)
$$

can be obtained analytically:

$$
\begin{aligned}
e^{-\lambda} & =1-\mathcal{C} \xi^{2}\left(5-3 \xi^{2}\right), \\
e^{\nu} & =(1-5 \mathcal{C} / 3) \cos ^{2} \phi
\end{aligned}
$$

Here $\xi=r / R$, and

$$
\begin{aligned}
\phi & =\left(w_{1}-w\right) / 2+\phi_{1}, \\
w & =\log \left[\xi^{2}-\frac{5}{6}+\sqrt{\frac{e^{-\lambda}}{3 \mathcal{C}}}\right], \\
\phi_{1} & =\phi\left(\xi^{2}=1\right)=\arctan \sqrt{\frac{\mathcal{C}}{3(1-2 \mathcal{C})}}, \\
w_{1} & =w\left(\xi^{2}=1\right) .
\end{aligned}
$$

Besides, the pressure can also be found exactly from the Tolman-Oppenheimer-Volkoff (TOV) equations [1, 2]:

$$
P=\frac{1}{4 \pi R^{2}}\left[\sqrt{3 \mathcal{C} e^{-\lambda}} \tan \phi-\frac{\mathcal{C}}{2}\left(5-3 \xi^{2}\right)\right]
$$

By virtue of (2.3), we have:

$$
\begin{aligned}
\xi & =\sqrt{1-\frac{\rho}{\rho_{0}}}, \\
e^{-\lambda} & =1-\mathcal{C}\left(1-\frac{\rho}{\rho_{0}}\right)\left(2+\frac{3 \rho}{\rho_{0}}\right) .
\end{aligned}
$$

The EOS of TVIIM is consequently given by:

$$
P=\frac{1}{4 \pi R^{2}}\left[\sqrt{3 \mathcal{C}-3 \mathcal{C}^{2}\left(1-\frac{\rho}{\rho_{0}}\right)\left(2+\frac{3 \rho}{\rho_{0}}\right)} \tan \phi-\mathcal{C}\left(1+\frac{3 \rho}{2 \rho_{0}}\right)\right],
$$

with

$$
\begin{aligned}
\phi= & \frac{1}{2} \log \left[\frac{1}{6}+\sqrt{\frac{1-2 \mathcal{C}}{3 \mathcal{C}}}\right]+\arctan \sqrt{\frac{\mathcal{C}}{3(1-2 \mathcal{C})}} \\
& -\frac{1}{2} \log \left[\frac{1}{6}-\frac{\rho}{\rho_{0}}+\sqrt{\frac{1}{3 \mathcal{C}}-\frac{1}{3}\left(1-\frac{\rho}{\rho_{0}}\right)\left(2+\frac{3 \rho}{\rho_{0}}\right)}\right]
\end{aligned}
$$

It is obvious that both $m_{c}(r)$ and the EOS for TVIIM depend on $M$ and $R$ (or any two independent parameters derivable from them). Thus, once these two parameters are known, the mass distribution inside the star and the EOS of the stellar matter are determined automatically. In the following discussion, we will make use of this salient feature of TVIIM as the foundation of the inversion scheme.

\section{APPROXIMATE MASS DISTRIBUTION}

Based on the the universality and the TVIIM discussed above, a feasible scheme is proposed here to infer the internal structure of a neutron star from its gravitational wave spectra. As the first step of the scheme, we consider the frequency of the leading (i.e. the least-damped) axial $w$-mode for TVIIM, $\omega_{1}^{(c)}=\omega_{1 r}^{(c)}-i \omega_{1 i}^{(c)}$, and plot the ratio $Q \equiv \omega_{1 r}^{(c)} / \omega_{1 i}^{(c)}$ against with the compactness in Fig. 目 We see that $\omega_{1 r}^{(c)} / \omega_{1 i}^{(c)}$ is in fact a monotonically function of 


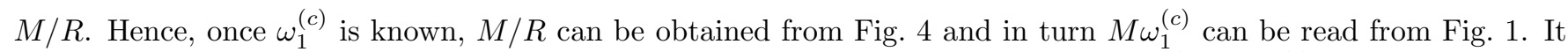
is then straightforward to find $M$ and $R$. Likewise, $M$ and $R$ can be inferred from $\omega_{2}^{(c)}$ and $\omega_{3}^{(c)}$, the frequencies of the second and the third leading modes, as $\omega_{2 r}^{(c)} / \omega_{2 i}^{(c)}$ and $\omega_{3 r}^{(c)} / \omega_{3 i}^{(c)}$ are also monotonic functions of the compactness $\mathcal{C}$ (see Fig. (4). However, the frequency $\omega_{1}^{(c)}$ is preferred in the subsequent discussion because it has the most sensitive dependence on $\mathcal{C}$.

As TVIIM provides a benchmark for other realistic stars, we expect that the eigenfrequency of the leading axial $w$-mode emitted from a realistic neuron star, $\omega_{1}=\omega_{1 r}-i \omega_{1 i}$, is close to that of a TVIIM star with the same mass $M$ and the same radius $R$. Therefore, we could go through the procedure outlined in the last paragraph with $\omega_{1}^{(c)}$ replaced by $\omega_{1}$ to obtain an estimate of the mass and the radius of the star in consideration. This first step in our scheme is analogous to the method proposed by Andersson and Kokkotas 22]. However, we will consider the frequencies of the non-leading modes and show that they can lead to much improved estimates of the mass, the radius and the EOS as well.

The mass distribution of a realistic neutron star is of course different from that of a TVIIM star. However, the difference is small. Considering the asymptotic behavior of the density around $r=0$ (see, e.g. [39]), we expand $m(r)$ as:

$$
m\left(r,\left\{\mu_{k}\right\}, M, R\right) \approx m_{0}(r, M, R)+\sum_{j=1}^{p-2} \mu_{j} m_{j}(r, M, R)
$$

where

$$
m_{j}(r, M, R)=\frac{15 M r}{2 R^{5}}\left(\frac{r}{R}\right)^{2 j}\left(r^{2}-R^{2}\right)^{2},
$$

$p \geq 2$, and $\mu_{j}(j=1,2, \ldots, p-2)$ are $p-2$ adjustable parameters to provide the best fit to $m\left(r,\left\{\mu_{k}\right\}, M, R\right)$. As the mass $M$ and the radius $R$ of the neutron star are not yet exactly known, they are also considered as free parameters. There are consequently $p$ free parameters to be determined from the QNM frequencies. Besides, as the zeroth order approximation to $m(r), m_{0}$ is well approximated by $m_{c}(r)$, which will be chosen to be the initial guess for $m_{0}$.

Generally speaking, we can approximate the configuration of a neutron star by choosing a set of $\mu_{j}, M$ and $R$ which "best fit" the exact one. Before working out the optimization process that determines these $p$ parameters, we briefly mention the physical significance of $m_{j}(r, M, R)$. It is clear that $m_{j}(r=R, M, R)=0$ for all $j$. Hence, $m_{j}$ does not affect the total mass of the neutron star while modifying the internal mass distribution. Furthermore, it can be shown that each $m_{j}$ has only one maximum value and the position of such maximum gets closer to the stellar surface as $j$ increases. This implies that $m_{j}$ with small $j$ has significant contribution to the core of the neutron star, while $m_{j}$ with large $j$ mainly affects the the mass distribution near the stellar surface. In addition, the central density of the neutron star depends only on $m_{0}$ and $m_{1}$. As a consequence, as far as the high density regime of the EOS is concerned, $m_{j}$ with smaller $j$ is likely to be more important. Lastly, the density distribution can be easily derived from the mass distribution,

$$
\rho\left(r, \mu_{k}, M, R\right)=\rho_{0}(r, M, R)+\sum_{j=1}^{p-2} \mu_{j} \rho_{j}(r, M, R),
$$

where

$$
\begin{gathered}
\rho_{0}(r, M, R)=\frac{15 M}{8 \pi R^{3}}\left[1-\left(\frac{r}{R}\right)^{2}\right] \\
\rho_{j}(r, M, R)=\frac{15 M}{8 \pi R^{7}}\left(\frac{r}{R}\right)^{2 j-2}\left(r^{2}-R^{2}\right)\left[(5+2 j) r^{2}-(1+2 j) R^{2}\right] .
\end{gathered}
$$

\section{THE INVERSION SCHEME}

\section{A. Optimal model}

Suppose that the mass and the radius of a star are known with certain accuracy from the the frequency of its leading mode, which are equal to $M_{0}$ and $R_{0}$, respectively. The exact values of these quantities, $M=M_{0}+M_{1}$ and 
$R=R_{0}+R_{1}$, and the mass distribution inside the star can be determined from the frequencies of QNMs of higher orders. Suppose $n(n>1)$ QNM frequencies of a neutron star are known and we are going to search for the optimal stellar model that yields the minimal deviation from the measured QNM frequencies. As discussed above, there are $p$ independent small parameters in the model, namely $\mu_{j}(j=1,2, \ldots, p-2), \mu_{p-1} \equiv M / R-M_{0} / R_{0} \equiv \mathcal{C}^{(1)}$, and $\mu_{p} \equiv M_{1}$. If all of these free parameters equal zero, the frequencies are denoted by $\omega_{q}^{(0)}$.

Based on the first order result of SCLPT [33], the corresponding first order change in the $q$-th $(q=1,2, \ldots, n)$ QNM frequencies $\omega_{q}$ is

$$
\omega_{q}^{(1)}=\sum_{j=1}^{p} \mu_{j} \frac{\mathrm{d} \omega_{q}}{\mathrm{~d} \mu_{j}}=\sum_{j=1}^{p} \mu_{j} c_{q j}
$$

which is close to the exact change:

$$
\Delta_{q}=\omega_{q}-\omega_{q}^{(0)}
$$

Explicit expressions for $c_{q j} \equiv \mathrm{d} \omega_{q} / \mathrm{d} \mu_{j}$ will be derived in the following section of this paper using SCLPT. By minimizing the square-deviation

$$
\begin{aligned}
\chi^{2} & =\sum_{q=1}^{n}\left|\Delta_{q}-\omega_{q}^{(1)}\right|^{2} \\
& =\sum_{q=1}^{n}\left(\Delta_{q} \bar{\Delta}_{q}-\Delta_{q} \sum_{j=1}^{p} \mu_{j} \bar{c}_{q j}-\bar{\Delta}_{q} \sum_{i=1}^{p} \mu_{i} c_{q i}+\sum_{i=1}^{p} \mu_{i} c_{q i} \sum_{j=1}^{p} \mu_{j} \bar{c}_{q j}\right),
\end{aligned}
$$

where the overbar indicates the complex conjugate of a variable, one can obtain a set of $\mu_{j}$ and hence an optimal approximate model for the observed neutron star. The necessary condition for $\chi^{2}$ to achieve a minimum is obviously:

$$
\frac{\partial \chi^{2}}{\partial \mu_{k}}=0
$$

for all $k$, leading to $n$ linear equations:

$$
\sum_{q=1}^{n}\left(\Delta_{q} \bar{c}_{q k}+\bar{\Delta}_{q} c_{q k}\right)=\sum_{j=1}^{p} \mu_{j} \sum_{q=1}^{n}\left(c_{q k} \bar{c}_{q j}+\bar{c}_{q k} c_{q j}\right) .
$$

This system of equations can be rewritten in the following matrix representation:

$$
\vec{b}=\mathbf{A} \vec{\mu},
$$

where $\mathbf{A}$ is a $p \times p$ square matrix, $\vec{\mu}$ is the column vector of $\left\{\mu_{j}\right\}$, and

$$
\begin{aligned}
b_{k} & =\sum_{q=1}^{n}\left(\Delta_{q} \bar{c}_{q k}+\bar{\Delta}_{q} c_{q k}\right), \\
A_{k j} & =\sum_{q=1}^{n}\left(c_{q k} \bar{c}_{q j}+\bar{c}_{q k} c_{q j}\right),
\end{aligned}
$$

for $k, j=1,2,3, \ldots, p$. Consequently, we can compute the best $\mu_{j}$ by solving the above matrix equation.

\section{B. Iterative scheme}

With the $p$ parameters $\left\{\mu_{j}\right\}$ obtained through the scheme sketched above, we can construct a new model star by incorporating these mass corrections into $m_{0}(r)$ and compute a new set of QNM frequencies for such a mass distribution. Again we can compare these QNM frequencies with the observed QNM frequencies $\omega_{q}$, and minimize the error $\chi^{2}$ by solving (4.6) to yield a new set of $\left\{\mu_{j}\right\}$. Through this iterative scheme, one can generate convergent sequences for the mass, the radius and the mass distribution. 


\section{Inversion of EOS}

The equilibrium configuration of a neutron star is given by the TOV equations [1, 2]:

$$
\begin{aligned}
\frac{\mathrm{d} m}{\mathrm{~d} r} & =4 \pi r^{2} \rho \\
\frac{\mathrm{d} \nu}{\mathrm{d} r} & =\frac{2 m+8 \pi r^{3} P}{r(r-2 m)} \\
\frac{\mathrm{d} P}{\mathrm{~d} r} & =-\frac{1}{2}(\rho+P) \frac{\mathrm{d} \nu}{\mathrm{d} r} .
\end{aligned}
$$

Here $\rho$ and $P$ are evaluated at a point $(r, \theta, \varphi)$. Once $m(r)$ is known, the density $\rho(r)$ can be found from (4.9). Combining (4.10) and (4.11) to yield a first-order differential equation for $P(r)$ :

$$
\frac{\mathrm{d} P}{\mathrm{~d} r}=-(\rho+P) \frac{m+4 \pi r^{3} P}{r(r-2 m)},
$$

one can obtain $P(r)$ by solving (4.12) with the boundary condition $P(r=R)=0$. Hence, the EOS can be straightforwardly inferred by comparing the two functions $\rho(r)$ and $P(r)$.

\section{SCLPT}

\section{A. Scaled Regge-Wheeler equation}

The starting point of the present inversion scheme is (4.1), which expresses the first-order shift in QNM frequencies as a linear sum over various perturbations $\left\{\mu_{j}\right\}$. To evaluate the coefficients $\left\{c_{q j}\right\}$ in (4.1), we have to invoke the theory of first-order SCLPT to find how various perturbations $\left\{\mu_{j}\right\}$ affect the QNM frequency [33].

First of all, we introduce the following scaled variables:

$$
\begin{aligned}
\tilde{r} & =\frac{r}{M}, \\
\tilde{r}_{*} & =\frac{r_{*}}{M}, \\
\tilde{m}(\tilde{r}) & =\frac{m(r)}{M}, \\
\tilde{P}(\tilde{r}) & =M^{2} P(r), \\
\tilde{\rho}(\tilde{r}) & =M^{2} \rho(r), \\
\tilde{\nu}(\tilde{r}) & =\nu(r), \\
\tilde{\omega} & =M \omega, \\
\tilde{V}\left(\tilde{r}_{*}\right) & =M^{2} V\left(r_{*}\right) .
\end{aligned}
$$

Here the tortoise coordinate $r_{*}$ is related to the circumferential radius $r$ by

$$
r_{*}=\int_{0}^{r} \mathrm{e}^{(-\nu+\lambda) / 2} \mathrm{~d} r
$$

and outside the star, the tortoise radial coordinate reduces to

$$
r_{*}=r+2 M \ln \left(\frac{r}{2 M}-1\right)+C^{\prime}
$$

where $C^{\prime}$ is a constant that can be obtained by matching (5.9) with (5.10) at $r=R$. Besides, we also let $\tilde{R} \equiv R / M$ and $\tilde{R}_{*} \equiv R_{*} / M$, where $R_{*}=r_{*}(r=R)$ is the tortoise radius of the star. In terms of these scaled variables, the neutron star Regge-Wheeler (NSRW) equation [39] governing the evolution of axial $w$-mode pulsation can be rewritten as [33]:

$$
\left[\frac{\mathrm{d}^{2}}{\mathrm{~d} \tilde{r}_{*}^{2}}+\tilde{\omega}^{2}-\tilde{V}\left(\tilde{r}_{*}\right)\right] \tilde{\psi}\left(\tilde{r}_{*}\right)=0
$$


where for $\tilde{r} \leq \tilde{R}$ the scaled Regge-Wheeler potential takes the form:

$$
\tilde{V}_{*}(\tilde{r})=\frac{e^{\tilde{\nu}}}{\tilde{r}^{3}}\left[l(l+1) \tilde{r}+4 \pi \tilde{r}^{3}(\tilde{\rho}-\tilde{P})-6 \tilde{m}(r)\right],
$$

and for $\tilde{r}>\tilde{R}$,

$$
\tilde{V}\left(\tilde{r}_{*}\right)=\left(1-\frac{2}{\tilde{r}}\right)\left[\frac{l(l+1)}{\tilde{r}^{2}}-\frac{6}{\tilde{r}^{3}}\right] .
$$

The scaled NSRW equation (5.11) is obviously more amenable to perturbative expansion since outside the star $\tilde{V}(\tilde{r})$ depends only on $\tilde{r}$. Hence, changes in the stellar mass do not directly affect the potential outside the star. Therefore, perturbation arising from the change in the stellar mass, which also leads to variations in mass density, pressure, and stellar radius, becomes localized inside the star. In the subsequent discussion, we will show that shifts in QNM frequencies can be expressed in terms of integrals with finite domains of integration.

\section{B. First order perturbation theory}

Consider an unperturbed neutron star which has a scaled circumferential (tortoise) radius $\tilde{R}_{0}\left(\tilde{R}_{* 0}\right)$, a scaled potential $\tilde{V}_{0}\left(\tilde{r}_{*}\right)$, and a scaled known mass distribution function $\tilde{m}_{0}(r)$. Note that all physical quantities are scaled with its own mass $M_{0}$. The unperturbed star (e.g. TVIIM) will be used to provide the zeroth-order approximation of a realistic star whose physical parameters are not exactly known and to be determined from the inversion scheme.

Analogous to other conventional perturbation theories, we will assume that the QNM wave function of the unperturbed star is known. If now the star is perturbed to a new configuration which is identical to that of the realistic star in consideration, the new potential function is given by:

$$
\tilde{V}\left(\tilde{r}_{*}\right)=\tilde{V}^{(0)}\left(\tilde{r}_{*}\right)+\tilde{V}^{(1)}\left(\tilde{r}_{*}\right) .
$$

As a consequence, the circumferential (tortoise) radius of the perturbed star is also modified such that, $\tilde{R}=\tilde{R}_{0}+\tilde{R}_{1}$ $\left(\tilde{R}_{*}=\tilde{R}_{* 0}+\tilde{R}_{* 1}\right)$. However, outside the star, $\tilde{V}$, as a function of $\tilde{r}$, remains unchanged.

Consider the $q$-th QNM of the unperturbed star, which has a scaled frequency $\tilde{\omega}_{q}^{(0)}$. From SCLPT we obtain the first-order shift in the scaled frequency $\tilde{\omega} \equiv M \omega$, which reads [35]:

$$
\tilde{\omega}_{q}^{(1)}=\frac{\left\langle\tilde{\psi}_{q}\left|\tilde{U}_{1}\right| \tilde{\psi}_{q}\right\rangle}{2 \tilde{\omega}_{q}^{(0)}\left\langle\tilde{\psi}_{q} \mid \tilde{\psi}_{q}\right\rangle}
$$

with

$$
\begin{gathered}
\left\langle\tilde{\psi}_{q}\left|\tilde{U}_{1}\right| \tilde{\psi}_{q}\right\rangle=\int_{0}^{\tilde{R}_{* 0}} \mathrm{~d} \tilde{r}_{*} \tilde{V}_{1}\left(\tilde{r}_{*}\right) \tilde{\psi}_{q}^{2}\left(\tilde{r}_{*}\right)+\tilde{\psi}_{q}^{2}\left(\tilde{R}_{* 0}\right)\left[\tilde{R}_{* 1} \frac{\mathrm{d} f_{q}^{(0)}}{\mathrm{d} \tilde{r}_{*}}-\tilde{R}_{1}\left(\frac{\partial f_{q}^{(+)}\left(\tilde{r}_{*}, \tilde{\omega}_{q}^{(0)}\right)}{\partial \tilde{r}}\right)\right]_{\tilde{r}_{*}=\tilde{R}_{* 0}}, \\
\left\langle\tilde{\psi}_{q} \mid \tilde{\psi}_{q}\right\rangle=\int_{0}^{\tilde{R}_{* 0}} \mathrm{~d} \tilde{r}_{*} \tilde{\psi}_{q}^{2}\left(\tilde{r}_{*}\right)+\frac{\tilde{\psi}_{q}^{2}\left(\tilde{R}_{* 0}\right)}{2 \tilde{\omega}_{q}^{(0)}}\left(\frac{\partial f_{q}^{(+)}\left(\tilde{R}_{* 0}, \tilde{\omega}\right)}{\partial \tilde{\omega}}\right)_{\tilde{\omega}=\tilde{\omega}_{q}^{(0)}},
\end{gathered}
$$

and $\tilde{\psi}_{q}\left(\tilde{r}_{*}\right)$ being the unperturbed wave function of the $q$-th QNM mode. Besides, $f_{q}^{(0)}\left(\tilde{r}_{*}\right)$ is the logarithmic derivative of the unperturbed wave function $\tilde{\psi}_{q}\left(\tilde{r}_{*}\right)$ that satisfies both the regular boundary condition at $\tilde{r}_{*}=0$ and the outgoing wave boundary condition at spatial infinity, while $f_{q}^{(+)}\left(\tilde{r}_{*}, \tilde{\omega}\right)$ is the logarithmic derivative of the outgoing wave solution of frequency $\tilde{\omega}$ to the scaled NSRW equation [33]. From (5.15), (5.16) and (5.17) it is then obvious that one has to evaluate the changes in the scaled potential, the scaled circumferential and tortoise radii of the star in order to find $\tilde{\omega}_{q}^{(1)}$. 


\section{The perturbed star}

The perturbed star is in fact the realistic star considered in the inversion scheme, whose scaled mass distribution $\tilde{m}(\tilde{r})$ and density distribution $\tilde{\rho}(\tilde{r})$ are expressed in terms of $\tilde{r}$ and its own exact compactness $\mathcal{C} \equiv M / R$ as follows:

$$
\begin{aligned}
\tilde{m}(\tilde{r}) & =\frac{m(r)}{M}=\tilde{m}_{0}(\tilde{r}, \mathcal{C})+\sum_{j=1}^{p-2} \mu_{j} \tilde{m}_{j}(\tilde{r}, \mathcal{C}) \\
\tilde{\rho}(\tilde{r}) & =M^{2} \rho(r)=\tilde{\rho}_{0}(\tilde{r}, \mathcal{C})+\sum_{j=1}^{p-2} \mu_{j} \tilde{\rho}_{j}(\tilde{r}, \mathcal{C}) .
\end{aligned}
$$

Here

$$
\begin{aligned}
\tilde{m}_{0}(\tilde{r}, \mathcal{C}) & =\frac{m_{0}(r, M, R)}{M}=\frac{1}{2}\left(5 \mathcal{C}^{3} \tilde{r}^{3}-3 \mathcal{C}^{5} \tilde{r}^{5}\right) \\
\tilde{m}_{j}(\tilde{r}, \mathcal{C}) & =\frac{m_{j}(r, M, R)}{M}=\frac{15}{2}(\mathcal{C} \tilde{r})^{1+2 j}\left(1-\mathcal{C}^{2} \tilde{r}^{2}\right)^{2} \\
\tilde{\rho}_{0}(\tilde{r}, \mathcal{C}) & =M^{2} \rho_{0}(r, M, R)=\frac{15}{8 \pi} \mathcal{C}^{3}\left(1-\mathcal{C}^{2} \tilde{r}^{2}\right) \\
\tilde{\rho}_{j}(\tilde{r}, \mathcal{C}) & =M^{2} \rho_{j}(r, M, R)=\frac{15}{8 \pi} \mathcal{C}^{2 j+1} \tilde{r}^{2 j-2}\left(\mathcal{C}^{2} \tilde{r}^{2}-1\right)\left[(5+2 j) \mathcal{C}^{2} \tilde{r}^{2}-(1+2 j)\right] .
\end{aligned}
$$

In order to compare the perturbed and unperturbed stars, we rewrite $\tilde{m}(\tilde{r})$ and $\tilde{\rho}(\tilde{r})$ as:

$$
\begin{aligned}
\tilde{m}(\tilde{r}) & =\tilde{m}^{(0)}(\tilde{r})+\tilde{m}^{(1)}(\tilde{r}), \\
\tilde{\rho}(\tilde{r}) & =\tilde{\rho}^{(0)}(\tilde{r})+\tilde{\rho}^{(1)}(\tilde{r}),
\end{aligned}
$$

with

$$
\begin{aligned}
& \tilde{m}^{(0)}(\tilde{r})=\tilde{m}_{0}\left(\tilde{r}, \mathcal{C}_{0}\right), \\
& \tilde{\rho}^{(0)}(\tilde{r})=\tilde{\rho}_{0}\left(\tilde{r}, \mathcal{C}_{0}\right), \\
& \tilde{m}^{(1)}(\tilde{r})=\sum_{j=1}^{p-1} \mu_{j} \tilde{m}_{j}\left(\tilde{r}, \mathcal{C}_{0}\right), \\
& \tilde{\rho}^{(1)}(\tilde{r})=\sum_{j=1}^{p-1} \mu_{j} \tilde{\rho}_{j}\left(\tilde{r}, \mathcal{C}_{0}\right), \\
& \tilde{m}_{p-1}(\tilde{r}) \equiv\left(\frac{\partial \tilde{m}_{0}}{\partial \mathcal{C}}\right)_{\mathcal{C}=\mathcal{C}_{0}}=\frac{15}{2}\left(\mathcal{C}_{0}^{2} \tilde{r}^{3}-\mathcal{C}_{0}^{4} \tilde{r}^{5}\right), \\
& \tilde{\rho}_{p-1}(\tilde{r}) \equiv\left(\frac{\partial \tilde{\rho}_{0}}{\partial \mathcal{C}}\right)_{\mathcal{C}=\mathcal{C}_{0}}=\frac{15}{8 \pi} \mathcal{C}_{0}^{2}\left(3-5 \mathcal{C}_{0}^{2} \tilde{r}^{2}\right),
\end{aligned}
$$

and $\mu_{p-1} \equiv \mathcal{C}-\mathcal{C}_{0}=\mathcal{C}^{(1)}$ as mentioned previously. $\tilde{m}^{(1)}(\tilde{r})$ and $\tilde{\rho}^{(1)}(\tilde{r})$ are considered as the first order changes in the mass and density distributions. Besides, it is straightforward to show that

$$
\begin{gathered}
\tilde{R}^{(1)} \equiv \frac{R}{M}-\frac{R_{0}}{M_{0}}=-\frac{\mathcal{C}^{(1)}}{\mathcal{C}^{(0)}}, \\
\tilde{R}_{*}^{(1)}=\tilde{R}^{(1)} \exp \left[\frac{\tilde{\nu}^{(0)}\left(\tilde{R}^{(0)}\right)-\tilde{\lambda}^{(0)}\left(\tilde{R}^{(0)}\right)}{2}\right]+\int_{0}^{\tilde{R}^{(0)}}\left[\frac{\tilde{\lambda}^{(1)}(\tilde{r})-\tilde{\nu}^{(1)}(\tilde{r})}{2}\right] \exp \left[\frac{\tilde{\lambda}^{(0)}(\tilde{r})-\tilde{\nu}^{(0)}(\tilde{r})}{2}\right] d \tilde{r} .
\end{gathered}
$$


These first-order quantities, including $\tilde{m}^{(1)}, \tilde{\rho}^{(1)}, \tilde{R}^{(1)}, \tilde{R}_{*}^{(1)}$, are the inputs to SCLPT. Once $\tilde{\omega}_{q}^{(1)}$ is found from the first-order SCLPT, the first order change in QNM frequency, $\omega^{(1)}$, can be obtained from the following relation:

$$
\begin{aligned}
\omega_{q}^{(1)} & =\frac{\tilde{\omega}_{q}^{(0)}+\tilde{\omega}_{q}^{(1)}}{M^{(0)}+M^{(1)}}-\frac{\tilde{\omega}_{q}^{(0)}}{M^{(0)}} \\
& =\frac{\tilde{\omega}_{q}^{(1)}}{M^{(0)}}-\frac{M^{(1)} \tilde{\omega}_{q}^{(0)}}{\left[M^{(0)}\right]^{2}}
\end{aligned}
$$

\section{Perturbed TOV equation}

The perturbation in $\tilde{m}^{(1)}(\tilde{r})$ (or $\tilde{\rho}^{(1)}(\tilde{r})$ ) of course leads to variations in pressure and metric coefficients, and in turn affects the potential $\tilde{V}$. So we need to consider the scaled TOV equations [32, 33],

$$
\begin{aligned}
\frac{d \tilde{m}}{d \tilde{r}} & =4 \pi \tilde{r}^{2} \tilde{\rho}(\tilde{r}), \\
\frac{d \tilde{\nu}}{d \tilde{r}} & =\frac{2 \tilde{m}(\tilde{r})+8 \pi \tilde{r}^{3} \tilde{P}(\tilde{r})}{\tilde{r}(\tilde{r}-2 \tilde{m}(\tilde{r}))} \\
\frac{d \tilde{P}}{d \tilde{r}} & =-\frac{1}{2}(\tilde{\rho}(\tilde{r})+\tilde{P}(\tilde{r})) \frac{d \tilde{\nu}}{d \tilde{r}}
\end{aligned}
$$

with $\tilde{\nu}=\nu$. Denoting the first order changes in $\tilde{P}$ and $\tilde{\nu}$ by $\tilde{P}^{(1)}$ and $\tilde{\nu}^{(1)}$, we can show from the scaled TOV equations that:

$$
\begin{aligned}
\frac{d \tilde{\nu}^{(1)}}{d \tilde{r}} & =\frac{1}{\left(\tilde{r}-2 \tilde{m}^{(0)}\right)^{2}}\left[\left(2+16 \pi \tilde{r}^{2} \tilde{P}^{(0)}\right) \tilde{m}^{(1)}+8 \pi \tilde{r}^{2}\left(\tilde{r}-2 \tilde{m}^{(0)}\right) \tilde{P}^{(1)}\right] \\
\frac{d \tilde{P}^{(1)}}{d \tilde{r}} & =-\frac{1}{2}\left[\left(\tilde{\rho}^{(1)}+\tilde{P}^{(1)}\right) \frac{d \tilde{\nu}^{(0)}}{d \tilde{r}}+\left(\tilde{\rho}^{(0)}+\tilde{P}^{(0)}\right) \frac{d \tilde{\nu}^{(1)}}{d \tilde{r}}\right] .
\end{aligned}
$$

Therefore, both $\tilde{P}^{(1)}$ and $\tilde{\nu}^{(1)}$ can be evaluated by numerically solving this pair of differential equations. Besides, from

$$
e^{-\tilde{\lambda}(\tilde{r})}=1-\frac{2 \tilde{m}(\tilde{r})}{\tilde{r}}
$$

we have

$$
\tilde{\lambda}^{(1)}=\frac{2 \tilde{m}^{(1)} \exp \left(\tilde{\lambda}^{(0)}\right)}{\tilde{r}}
$$

\section{E. Perturbed potential}

Upon the changes in mass and density functions, the scaled $\tilde{V}_{r w}$ varies in accordance with (5.14). However, $\tilde{V}$ is an explicit function of $\tilde{r}$, instead of $\tilde{r}_{*}$. In order to express $\tilde{V}$ as a function of $\tilde{r}_{*}$ such that our perturbation scheme is applicable, we have to regard $\tilde{r}$ as a function of $\tilde{r}_{*}$ and in turn obtain the implicit function $\tilde{V}\left(\tilde{r}\left(\tilde{r}_{*}\right)\right)$. In the absence of perturbation, $\tilde{r}=\tilde{r}^{(0)}\left(\tilde{r}_{*}\right)$ is determined from the defining equation of the tortoise coordinate:

$$
\tilde{r}_{*}=\int_{0}^{\tilde{r}^{(0)}\left(\tilde{r}_{*}\right)} \exp \left\{\left[-\tilde{\nu}^{(0)}(\tilde{r})+\tilde{\lambda}^{(0)}(\tilde{r})\right] / 2\right\} d \tilde{r}
$$

With the introduction of $\tilde{m}^{(1)}$ and $\tilde{\rho}^{(1)}$, the scaled circumferential radius is modified such that $\tilde{r}=\tilde{r}^{(0)}\left(\tilde{r}_{*}\right)+\tilde{r}^{(1)}\left(\tilde{r}_{*}\right)$, where $\tilde{r}^{(1)}\left(\tilde{r}_{*}\right)$ is defined via the following equation:

$$
\tilde{r}_{*}=\int_{0}^{\tilde{r}^{(0)}\left(\tilde{r}_{*}\right)+\tilde{r}^{(1)}\left(\tilde{r}_{*}\right)} \exp \left\{\left[\tilde{\lambda}^{(0)}(\tilde{r})+\tilde{\lambda}^{(1)}(\tilde{r})-\tilde{\nu}^{(0)}(\tilde{r})-\tilde{\nu}^{(1)}(\tilde{r})\right] / 2\right\} d \tilde{r}
$$


Neglecting second order terms, we can find $\tilde{r}^{(1)}$ in terms of $\tilde{r}_{*}$ :

$$
\tilde{r}^{(1)}\left(\tilde{r}_{*}\right)=\exp \left[\frac{\tilde{\nu}^{(0)}\left(\tilde{R}^{(0)}\right)-\tilde{\lambda}^{(0)}\left(\tilde{R}^{(0)}\right)}{2}\right] \int_{0}^{\tilde{r}^{(0)}\left(\tilde{r}_{*}\right)}\left[\frac{\tilde{\nu}^{(1)}(\tilde{r})-\tilde{\lambda}^{(1)}(\tilde{r})}{2}\right] \exp \left[\frac{\tilde{\lambda}^{(0)}(\tilde{r})-\tilde{\nu}^{(0)}(\tilde{r})}{2}\right] d \tilde{r} .
$$

By virtue of (5.14), we have

$$
\begin{aligned}
\tilde{V}^{(1)}\left(\tilde{r}_{*}\right) & =\tilde{V}\left(\tilde{r}^{(1)}+\tilde{r}^{(0)}\right)-\tilde{V}^{(0)}\left(\tilde{r}^{(0)}\right) \\
& =\left[\tilde{V}\left(\tilde{r}^{(0)}\right)-\tilde{V}^{(0)}\left(\tilde{r}^{(0)}\right)\right]+\tilde{r}^{(1)} \frac{d \tilde{V}^{(0)}}{d \tilde{r}^{(0)}},
\end{aligned}
$$

and from (5.12) explicit expressions for the terms in the right hand side of (5.42) can be found:

$$
\begin{gathered}
\frac{d \tilde{V}^{(0)}}{d \tilde{r}^{(0)}}=\tilde{V}^{(0)} \frac{\partial \tilde{\nu}^{(0)}}{\partial \tilde{r}^{(0)}}+e^{\tilde{\nu}^{(0)}}\left\{-\frac{2 l(l+1)}{\left(\tilde{r}^{(0)}\right)^{3}}+4 \pi\left(\frac{d \tilde{\rho}^{(0)}}{d \tilde{r}^{(0)}}-\frac{d \tilde{P}^{(0)}}{d \tilde{r}^{(0)}}\right)-\frac{6\left[4 \pi\left(\tilde{r}^{(0)}\right)^{3} \tilde{\rho}^{(0)}-3 \tilde{m}^{(0)}\right]}{\left(\tilde{r}^{(0)}\right)^{4}}\right\}, \\
\tilde{V}\left(\tilde{r}^{(0)}\right)-\tilde{V}^{(0)}\left(\tilde{r}^{(0)}\right)=\tilde{\nu}^{(1)} \tilde{V}^{(0)}\left(\tilde{r}^{(0)}\right)+e^{\tilde{\nu}^{(0)}}\left[4 \pi\left(\tilde{\rho}^{(1)}-\tilde{P}^{(1)}\right)-\frac{6 \tilde{m}^{(1)}}{\left(\tilde{r}^{(0)}\right)^{3}}\right] .
\end{gathered}
$$

The results obtained above together with the first order SCLPT lead explicit expressions for $c_{q j}$ in (4.1).

\section{NUMERICAL RESULTS}

In Figs. [5-10] we show numerical results obtained from the inversion scheme developed in the present paper for six different realistic neutron stars with a common compactness of 0.28 . To gauge the accuracy of the scheme, we have used four different combinations of QNM frequencies: (i) $\omega_{1}$; (ii) $\omega_{1}$ and $\omega_{2}$; (iii) $\omega_{1}, \omega_{2}$ and $\omega_{3}$; and (iv) $\omega_{1}$ and $\omega_{\text {II }}$. Here $\omega_{1}, \omega_{2}, \omega_{3}, \ldots$ are ordered in increasing frequencies, and $\omega_{\text {II }}$ is the frequency of a $w_{\text {II }}$ mode [40]. In all cases the number of parameters $p$ was chosen to be twice the number of QNMs (i.e. $2 n$ ). It is clearly seen from these figures that (i) the results obtained from using only the frequency of the least damped mode, $\omega_{1}$ (empty symbols), can nicely reproduce the EOS except at the high density regime; (ii) the results obtained from a combination of $\omega_{1}$ and $\omega_{2}$ (grey symbols) are readily improved; (iii) using all three frequencies $\omega_{1}, \omega_{2}$ and $\omega_{3}$ (dark symbols) indeed yields a perfect match with the exact values; and (iv) the results obtained from the combination of $\omega_{1}$ and $\omega_{\text {II }}$ (crosses) are slightly less accurate than those from the combination of $\omega_{1}$ and $\omega_{2}$.

In Fig. 2] we compare these six EOSs and demonstrate that it suffices to use only two frequencies $\left(\omega_{1}\right.$ and $\left.\omega_{2}\right)$ to accurately reproduce and distinguish these EOSs. In Table I we tabulate the exact values of $M$ and $R$ and those obtained from our scheme using one or two QNMs for stars constructed with these EOSs. The result is truly encouraging, especially for the mass, which is very close to the exact value in all cases. Despite that the computed radius might deviate from the exact one by a few percent in some cases, the result is in fact very accurate taking into account of the fact that the mass density near the surface of realistic neutron stars is usually very low. Hence, we conclude that the frequencies of two leading $w$-mode oscillations can readily lead to accurate determination of $M, R$ and EOS.

In each inversion process the number of parameters $p$ is less than or equal to twice the number of QNMs used (i.e. $2 n)$. This can be easily understood as each QNM frequency carries two data, namely the pulsation and decay rates. As a result, at most $2 n$ parameters can be determined from the inversion process. On the other hand, as shown in Fig. [1] where the inversion scheme is applied to an APR1 star with three known QNMs, the case $p=6$ obviously yields the best result. Therefore, for a fixed number of QNMs, the choice $p=2 n$ seems to be the optimal one and this remark is supported by our numerical results.

The stability of the inversion process has been investigated by introducing artificial noises into the real and imaginary parts of QNM frequencies. In each inversion process the radius of the star in consideration, which was also an unknown to be determined from the process, was divided into a fixed number of equal partitions. The values of the radius, density, and pressure at each of these small partitions were in turn obtained from the inversion scheme. After gathering the data, we averaged them over a number of simulations in which random noises were superimposed on the values of QNM frequencies, and evaluated the respective error bars. Fig. 12 shows the result for an APR1 star with two approximately known QNM frequencies, $\omega_{1}$ and $\omega_{2}$, and four parameters were used in the inversion. The random noise introduced was within $\pm 5 \%$ of the exact value. The grey circles (with error bars) denote the average values 
of 12 simulations, which are fairly accurate. This strongly suggests that the inversion scheme proposed here is still feasible even if gravitational signals are blurred by experimental noises. However, there was a need for caution to be exercised in inverting noise-polluted QNM frequencies. In some cases the result obtained might explicitly violate the thermodynamic stability condition $\mathrm{d} \rho / \mathrm{d} p$. We have in fact discarded such ill-defined data in the inversion scheme mentioned above.

Lastly, to further demonstrate the robustness of the inversion scheme, we have also applied our scheme to infer the EOS of a SQS [13]. The EOS of quark matter is given by the MIT bag model [1]:

$$
P=(\rho-4 B) / 3
$$

where the value of the bag constant $B$ is $57 \mathrm{MeV} \mathrm{fm}^{-3}$. The crust of the SQS star, a thin layer of normal matter obeying the Baym-Pethick-Sutherland EOS [3] , is supported by Coulomb forces above the quark matter core [13]. The EOS for quark matter is rather exotic and the density profile of such a star is even discontinuous across the inner boundary of the crust. However, as shown in Fig. 13, the inversion scheme still works nicely as long as four QNMs are used. It is then obvious that the current inversion scheme outperform the one proposed by Lindblom, which is limited to barotropic type EOS [34].

\section{DETECTION OF QNMS}

In the above discussion we show how the internal structure of a neutron star can be systematically determined from several of its QNM frequencies. Like all other studies in the area of gravitational-wave asteroseismology (see e.g. [21, 22, 23, 24, 25, 26, 27, 28, 29]), our method depends crucially on reliable data of gravitational wave signals, which have not yet been available. Therefore, the realization of the present scheme hinges on the successful operation of various existing gravitational-wave detectors (such as LIGO, VIRGO, GEO600), and also the development in the design and construction of new gravitational-wave detectors in the future. In the following discussion, we consider the possibility of detecting QNMs of neutron stars based on analysis outlined in Ref. 24].

The detection of a QNM signal, an exponentially decaying sinusoidal wave with frequency $f=\omega_{r} /(2 \pi)$ and decay time $\tau=1 / \omega_{i}$, depends on the distance $r$ between the emitter (the neutron star) and the observer (the earth), the energy $E_{\mathrm{gw}}$ channelled in such a QNM, and of course the sensitivity of the gravitational-wave detector, which is measured in terms of its noise power spectral density $S_{n}(f)$ (see Fig. 14). A strong signal is associated with a large signal-to-noise ratio given by [24]:

$$
\left(\frac{S}{N}\right)^{2}=\frac{4 Q^{2}}{1+4 Q^{2}} \frac{A^{2} \tau}{2 S_{n}}
$$

where the quality factor $Q=\pi f \tau$, and the amplitude of such a gravitational wave signal [24]:

$$
A \sim 2.4 \times 10^{-20}\left(\frac{E_{\mathrm{gw}}}{10^{-6} \mathrm{M}_{\odot} c^{2}}\right)^{1 / 2}\left(\frac{10 \mathrm{kpc}}{r}\right)\left(\frac{1 \mathrm{kHz}}{f}\right)\left(\frac{1 \mathrm{~ms}}{\tau}\right)^{1 / 2} .
$$

The fractional statistical errors in the determination of the frequency and the decay time, respectively denoted by $\sigma_{f} / f$ and $\sigma_{\tau} / \tau$, are estimated to be [24]:

$$
\begin{gathered}
\frac{\sigma_{f}}{f} \simeq 0.0042 P^{-1} \sqrt{\frac{1-2 Q^{2}+8 Q^{4}}{4 Q^{4}}}\left(\frac{\tau}{1 \mathrm{~ms}}\right)^{-1}, \\
\frac{\sigma_{\tau}}{\tau} \simeq 0.013 P^{-1} \sqrt{\frac{10+8 Q^{2}}{Q^{2}}}\left(\frac{f}{1 \mathrm{kHz}}\right),
\end{gathered}
$$

with

$$
P^{-1}=\left(\frac{E_{\mathrm{gw}}}{10^{-6} \mathrm{M}_{\odot} c^{2}}\right)^{-1 / 2}\left(\frac{r}{10 \mathrm{kpc}}\right)\left(\frac{S_{n}^{1 / 2}}{10^{-23} \mathrm{~Hz}^{-1 / 2}}\right) .
$$

Both LIGO and VIRGO have a broad-band sensitivity in the hundred-Hertz range where $S_{n}^{1 / 2} \sim 10^{-23} \mathrm{~Hz}^{-1 / 2}$ (see Fig. 14). It is then obvious that these first generation detectors could not accurately locate $w$-mode QNMs 
of neutron stars in our galaxy, which are characterized by the following typical values: $f \sim 10 \mathrm{kHz}, \tau \sim 0.02 \mathrm{~ms}$, $E_{\mathrm{gw}} \sim 10^{-6} \mathrm{M}_{\odot} c^{2}$ and $r \sim 10 \mathrm{kpc}$ [24]. In fact, it is generally believed that the sensitivities of the first generation detectors are not sufficient to carry out quantitative measurements.

LIGO-II, a second generation gravitational-wave detector, is aimed at operation in the hundred-Hertz range near the standard quantum limit (SQL) and has a lower noise level given approximately by [41]:

$$
\frac{S_{n}(f)}{\mathrm{Hz}^{-1}}=10^{-49}\left[x^{-4.14}-5 x^{-2}+\frac{(111)\left(2-2 x^{2}+x^{4}\right)}{2+x^{2}}\right],
$$

with $x=f /(215 \mathrm{~Hz})$. In spite of the improved sensitivity, as shown in Table $\Pi$ where $E_{\mathrm{gw}}=10^{-6} \mathrm{M}_{\odot} c^{2}$ and $r=10 \mathrm{kpc}$ are assumed, LIGO-II is not yet sensitive enough to locate $w$-mode QNMs of neutron stars in our own galaxy. On the other hand, it is worthwhile to note that SQL can in fact be overcome by utilizing quantum nondemolition (QND) devices [42]. The design of LIGO-III, a third generation gravitational-wave detector, is currently under intense discussion. Various strategies that can beat the gravitational-wave SQL by an arbitrarily large amount, over an arbitrarily wide range of frequencies have been proposed and analyzed [43, 44].

In the present paper we consider specifically another third generation gravitational-wave detector, EURO (European Gravitational Wave Observatory), proposed by a group of European scientists in 2000, aims to make quantitative surveys of gravitational-wave signals over 4 decades of frequency, namely from $1 \mathrm{~Hz}$ to tens of $\mathrm{kHz}$ [29, 41]. As stated in the proposal [41], "EURO can do asteroseismology on neutron stars by resolving the frequencies of the dominant normal modes of vibration". In the proposal two possible configurations of EURO were discussed. In the first configuration, EURO, the sensitivity in the high frequency range is still limited by the photon shot noises, with a noise level given by [41]:

$$
\frac{S_{n}(f)}{\mathrm{Hz}^{-1}}=10^{-50}\left[\frac{3.6 \times 10^{9}}{f^{4}}+\frac{1.3 \times 10^{5}}{f^{2}}+1.3 \times 10^{-3} f_{k}\left(1+\frac{f^{2}}{f_{k}^{2}}\right)\right],
$$

where the knee-frequency $f_{k}=1000 \mathrm{~Hz}$. As shown in Fig. 14 the noise level of this configuration in the kHz range is two orders less than that of LIGO-II. With this detector $w$-mode gravitational wave signals emitted from neutron stars within our galaxy can be detected with error bound of a few percent. Therefore, the inversion scheme outlined above will become viable once EURO operates as proposed.

In the second configuration, referred to as EURO (xylophone) in the present paper, the shot noise limit in the high-frequency regime is beaten by parallel operation of several narrow-banded cryogenic interferometers comprising a "gravitational-wave xylophone" [29, 41]. The noise level of such a device is approximately given by [1]:

$$
\frac{S_{n}(f)}{\mathrm{Hz}^{-1}}=10^{-50}\left[\frac{3.6 \times 10^{9}}{f^{4}}+\frac{1.3 \times 10^{5}}{f^{2}}\right],
$$

which can be obtained by removing the last term (i.e. the shot-noise term) in (7.7). As can be inferred from Fig. 14] Table [I] and also the following scaling relations [24]:

$$
\begin{aligned}
& \left(\frac{S}{N}\right)^{2} \propto \frac{E_{\mathrm{gw}}}{r^{2}}, \\
& \left(\frac{\sigma_{f}}{f}\right) \propto \frac{r}{\sqrt{E_{\mathrm{gw}}}}, \\
& \left(\frac{\sigma_{\tau}}{\tau}\right) \propto \frac{r}{\sqrt{E_{\mathrm{gw}}}},
\end{aligned}
$$

the advent of the detector EURO (xylophone) will enable observation of gravitational waves from extra-galactic neutron stars and black holes. We therefore expect that the inversion scheme developed in the present paper can reveal fruitful information about the internal structure of neutron stars from the data gathered by these third generation gravitational-wave detectors.

\section{CONCLUSION}

Despite the mass of some neutron star binaries can be inferred from their orbital periods, as yet there is no generic method to determine their radii except for a few cases where data of gravitational red-shift are available [6, 7]. In the present paper we have proposed a robust inversion scheme to determine the mass, the radius, the mass distribution 
and the EOS of a neutron star from its gravitational wave spectra, which could be obtained by the matched filtering method (see, e.g. [24]). We expect that our scheme could operate in conjunction with gravitational-wave detectors available in one or two decades, e.g. LIGO-III, EURO and EURO (xylophone), to probe the interior of neutron stars. As clearly shown in Fig. 22 an inversion scheme using two leading QNM frequencies can readily discriminate between any two smooth EOSs considered there. The method developed in the present paper in fact makes the construction of such gravitational-wave detectors more rewarding.

In reality, gravitational signals are inevitably contaminated by noises of various kinds, including thermal, quantum and gravity-gradient noises (see, e.g. [24, 43, 44, 45, 46, 47] and references therein). Analogous to other fields of astronomy, observational errors introduced by noises are deemed the major hurdle to the implementation of the inversion scheme proposed here. In order to infer the fine internal structure of neutron stars, theoretical and experimental efforts must be done to minimize the noise level, and sophisticated data acquisition skills have to be developed to distinguish signals from noises [41, 43, 44]. On the other hand, as demonstrated in Fig. 12 our method remains accurate and stable against contamination of data created by noises. The construction and operation of advanced gravitational-wave detectors that can provide higher detection sensitivities and resolution power will strongly boost the progress in gravitational-wave asteroseismology [41, 43, 44]. The proposal in the current paper will then become a powerful tool. As predicted in Refs. [29, 41], the prospect of gravitational-wave asteroseismology is bright.

\section{Acknowledgments}

We thank K Young, WM Suen and LM Lin for discussions. Our work is supported in part by the Hong Kong Research Grants Council (Grant No: 401905) and a direct grant (Project ID: 2060260) from the Chinese University of Hong Kong. 
[1] J. R. Oppenheimer and G. M. Volkoff, Phys. Rev. 55, 374 (1939).

[2] R. C. Tolman, Phys. Rev. 55, 364 (1939).

[3] N. K. Glendenning, Compact Stars - Nuclear Physics, Particle Physics, and General Relativity (Springer, NY, 1997).

[4] A. Akmal, V. R. Pandharipande, and D. G. Ravenhall, Phys. Rev. C 58, 1804 (1998).

[5] J. M. Lattimer and M. Prakash, Astrophys. J. 550, 426 (2001).

[6] F. Özel, Nature 441, 1115 (2006).

[7] J. Cottam, F. Paerels, and M. Mendez, Nature 420, 51 (2002).

[8] C. Alcock, E. Farhi, and A. Olinto, Phys. Rev. Lett. 57, 2088 (1986).

[9] N. K. Glendenning, Phys. Rev. Lett. 63, 2629 (1989).

[10] K. S. Cheng, Z. G. Dai, D. M. Wei, and T. Lu, Science 280, 407 (1998).

[11] A. Chodos, R. L. Jaffe, K. Johnson, C. B. Thorne, and V. F. Weisskopf, Phys. Rev. D 9, 3471 (1974).

[12] E. Witten, Phys. Rev. D 30, 272 (1984).

[13] C. Alcock, C. E. Farhi, and A. Olinto, Astrophys. J. 310, 261 (1986).

[14] M. Prakash, E. Baron, and M. Prakash, Phys. Lett. B 243, 175 (1990).

[15] S. Hughes, Ann. Phys. 303, 142 (2003).

[16] J. W. Mason, ed., Astrophysics Update (Springer-Praxis, 2004), vol. I, pp. 281-310.

[17] K. Belczynski, V. Kalogera, and T. Bulik, Astrophys. J. 572, 407 (2002).

[18] V. Kalogera et al., Astrophys. J. 601, L179 (2004).

[19] L. Lindblom, B. J. Owen, and S. M. Morsink, Phys. Rev. Lett. 80, 4843 (1998).

[20] C. L. Fryer, D. E. Holz, and S. A. Hughes, Astrophys. J. 565, 430 (2002).

[21] N. Andersson and K. D. Kokkotas, Phys. Rev. Lett. 77, 4134 (1996).

[22] N. Andersson and K. D. Kokkotas, MNRAS 299, 1059 (1998).

[23] O. Benhar, E. Berti, and V. Ferrari, MNRAS 310, 797 (1999).

[24] K. D. Kokkotas, T. A. Apostolatos, and N. Andersson, MNRAS 320, 307 (2001).

[25] G. Miniutti, J. A. Pons, E. Berti, L. Gualtieri, and V. Ferrari, MNRAS 338, 389 (2003).

[26] H. Sotani and T. Harada, Phys. Rev. D 68, 024019 (2003).

[27] O. Benhar, V. Ferrari, and L. Gualtieri, Phys. Rev. D 70, 124015 (2004).

[28] Y. Kojima and K. Sakata, Prog. Theor. Phys. 108, 801 (2002).

[29] N. Andersson and G. L. Comer, Phys. Rev. Lett. 87, 241101 (2001).

[30] E. S. C. Ching, P. T. Leung, A. M. van den Brink, W. M. Suen, S. S. Tong, and K. Young, Rev. Mod. Phys. 70, 1545 (1998).

[31] K. D. Kokkotas and B. G. Schmidt, Living Rev. Rel. 2, 2 (1999).

[32] L. K. Tsui and P. T. Leung, MNRAS 357, 1029 (2005).

[33] L. K. Tsui and P. T. Leung, Astrophys. J. 631, 495 (2005).

[34] L. Lindblom, Astrophys. J. 398, 569 (1992).

[35] L. K. Tsui and P. T. Leung, Phys. Rev. Lett. 95, 151101 (2005).

[36] V. Pandharipande, Nucl. Phys A 174, 641 (1971).

[37] V. Pandharipande, Nucl. Phys A 178, 123 (1971).

[38] R. B. Wiringa, V. Fiks, and A. Fabrocini, Phys. Rev. C 38, 1010 (1988).

[39] S. Chandrasekhar and V. Ferrari, Proc. R. Soc. A 432, 247 (1991).

[40] M. Leins, H. P. Nollert, and M.H. Soffel, Phys. Rev. D 48, 3467 (1993).

[41] EURO homepage http://www.astro.cf.ac.uk/geo/euro

[42] V. B. Braginsky and Y. I. Vorontsov, Sov. Phys. Usp. 17, 644 (1975).

[43] H. J. Kimble, Y. Levin, A. B. Matsko, K. S. Thorne, and S. P. Vyatchanin, Phys. Rev. D 65, 022002 (2001).

[44] P. Purdue and Y. Chen, Phys. Rev. D 66, 122004 (2002).

[45] Y. T. Liu and K. S. Thorne, Phys. Rev. D 62, 122002 (2000).

[46] K. S. Thorne and C. J. Winstein, Phys. Rev. D 60, 082001 (1999).

[47] D. H. Santamore and Y. Levin, Phys. Rev. D 64, 042002 (2001). 


\begin{tabular}{|c|c|c|}
\hline EOS & $M\left(10^{33} \mathrm{gm}\right)$ & $R(\mathrm{~km})$ \\
\hline \hline APR1 & $4.460 / 4.475 / 4.460$ & $11.83 / 12.12 / 11.70$ \\
\hline APR2 & $4.173 / 4.184 / 4.173$ & $11.07 / 11.26 / 10.91$ \\
\hline AU & $3.843 / 3.862 / 3.843$ & $10.19 / 10.61 / 10.08$ \\
\hline A & $3.289 / 3.282 / 3.289$ & $8.723 / 8.529 / 8.564$ \\
\hline C & $2.791 / 2.788 / 2.791$ & $7.404 / 7.185 / 7.216$ \\
\hline UT & $3.654 / 3.651 / 3.655$ & $9.691 / 9.745 / 9.664$ \\
\hline
\end{tabular}

TABLE I: The mass $M$ and the radius $R$ of neutron stars constructed with different EOSs are compared with the corresponding values obtained from inversion using one or two QNMs. In each entry the values are obtained from TOV equation/inversion using $\omega_{1} /$ inversion using $\omega_{1}$ and $\omega_{2}$ respectively.

\begin{tabular}{|c|c|c|c|c|c|c|}
\hline Detector & $f(\mathrm{kHz})$ & $\tau(\mathrm{ms})$ & {$\left[S_{n}(f)\right]^{1 / 2}\left(\mathrm{~Hz}^{-1 / 2}\right)$} & $(S / N)^{2}$ & $\sigma_{f} / f$ & $\sigma_{\tau} / \tau$ \\
\hline \hline LIGO II & 7.320 & 0.065 & $1.13 \times 10^{-22}$ & $3.76 \times 10^{-1}$ & 0.99 & 3.8 \\
& 12.76 & 0.037 & $1.98 \times 10^{-22}$ & $4.06 \times 10^{-2}$ & 3.07 & 11.6 \\
\hline EURO & 7.320 & 0.065 & $8.42 \times 10^{-25}$ & $6.80 \times 10^{3}$ & $7.4 \times 10^{-3}$ & $2.8 \times 10^{-2}$ \\
& 12.76 & 0.037 & $1.46 \times 10^{-24}$ & $7.45 \times 10^{2}$ & $2.3 \times 10^{-2}$ & $8.5 \times 10^{-2}$ \\
\hline EURO & 7.320 & 0.065 & $4.93 \times 10^{-27}$ & $1.99 \times 10^{8}$ & $4.3 \times 10^{-5}$ & $1.7 \times 10^{-4}$ \\
(xylophone) & 12.76 & 0.037 & $2.83 \times 10^{-27}$ & $1.99 \times 10^{8}$ & $4.4 \times 10^{-5}$ & $1.7 \times 10^{-4}$ \\
\hline
\end{tabular}

TABLE II: The noise amplitude spectral density $\left[S_{n}(f)\right]^{1 / 2}\left(\mathrm{~Hz}^{-1 / 2}\right)$, the signal to noise ratio $(S / N)^{2}, \sigma_{f} / f$ and $\sigma_{\tau} / \tau$ are tabulated for LIGO II, EURO and EURO (xylophone) gravitational-wave detectors. Here the frequency and damping time of the two leading axial $w$-modes of an APR1 star with $\mathcal{C}=0.28$ are considered, which are also used as the input in Fig. 12 and $E_{\mathrm{gw}}=10^{-6} \mathrm{M}_{\odot} c^{2}$ and $r=10 \mathrm{kpc}$ are assumed. 


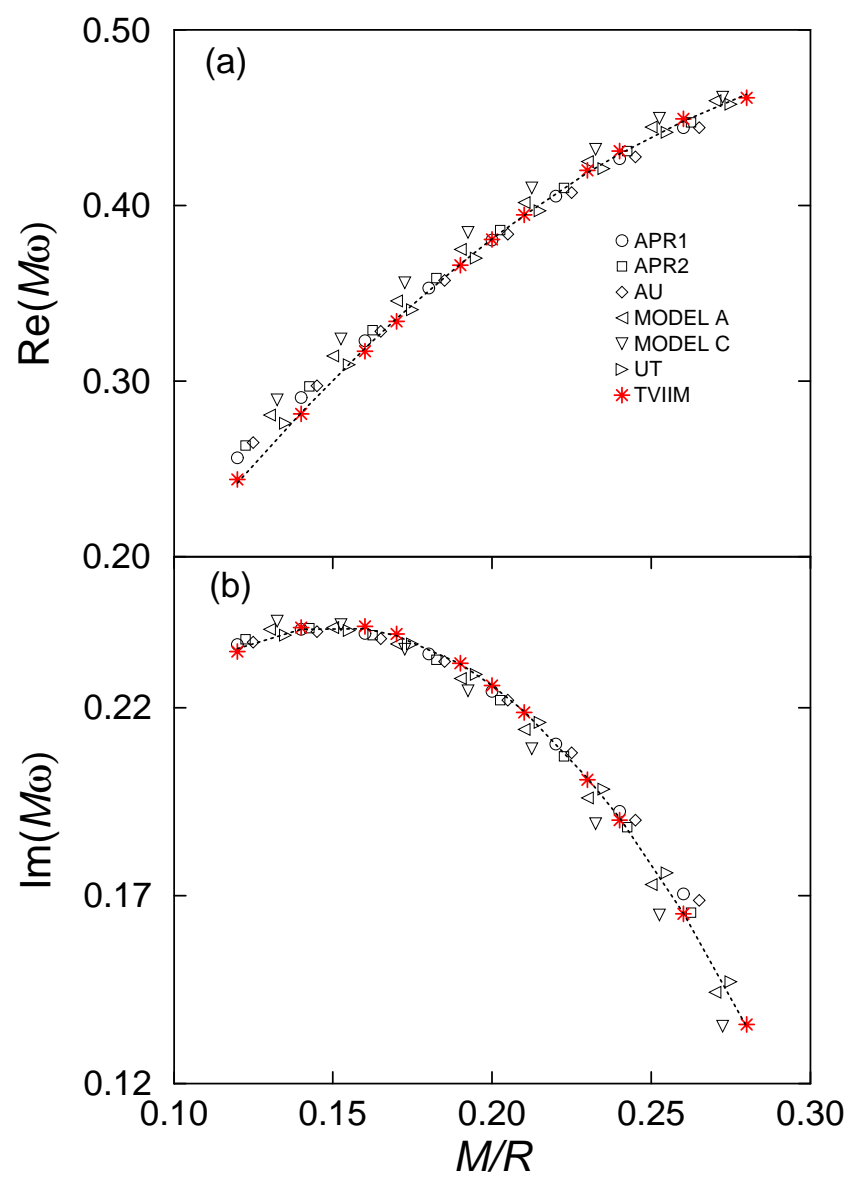

FIG. 1: The real and imaginary parts of $M \omega$ for the least-damped axial $w$-mode of six realistic stars (unfilled symbols) and TVIIM (stars) are shown as a function of $M / R$ in panels (a) and (b) respectively. The dotted line represents the best quadratic fit to those of TVIIM.

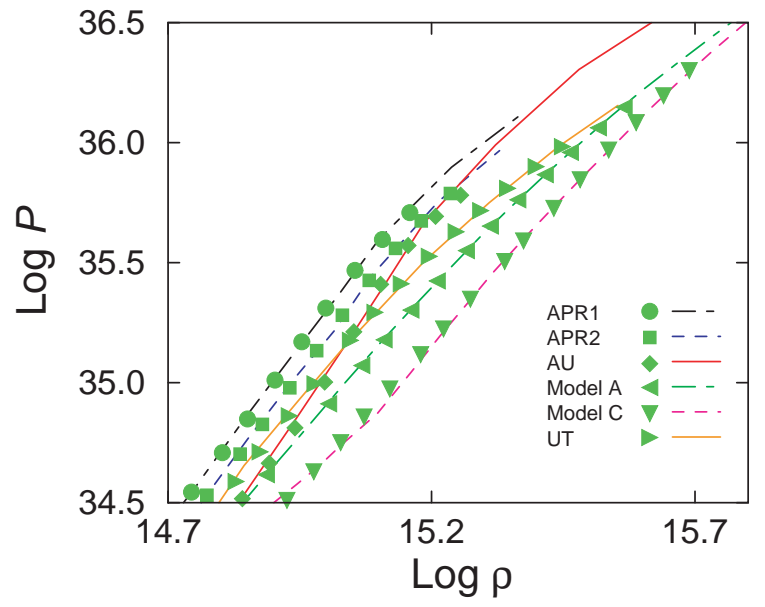

FIG. 2: Six different EOSs are shown by lines of different formats. Grey symbols are the corresponding values obtained from inversion scheme using $\omega_{1}$ and $\omega_{2}$ for stars with $\mathcal{C}=0.28$. Here $P$ and $\rho$ are in cgs units. 


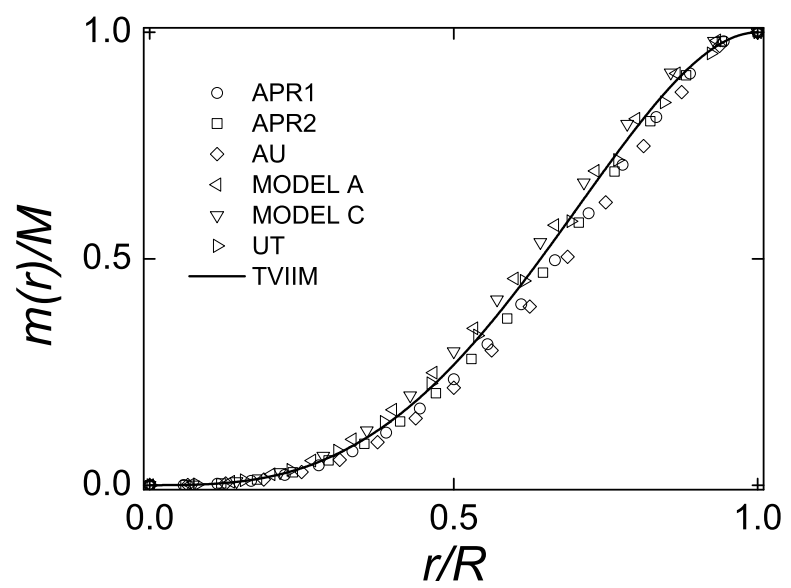

FIG. 3: The scaled mass distributions $m(r) / M$ of various realistic neutron stars and TVIIM with a common compactness $\mathcal{C}=0.28$ are plotted against $r / R$.

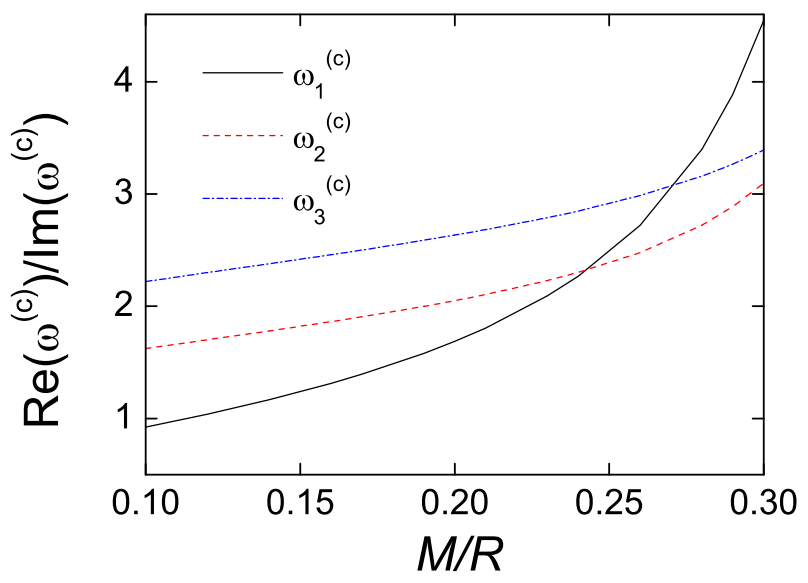

FIG. 4: $\operatorname{Re}\left(\omega^{(c)}\right) / \operatorname{Im}\left(\omega^{(c)}\right)$ for the three leading modes of TVIIM star (i) $\omega_{1}^{(c)}$ (solid-line); (ii) $\omega_{2}^{(c)}$ (dashed-line) and (iii) $\omega_{3}^{(c)}$ (dot-dashed-line) are plotted against the compactness $M / R$. 
(a)
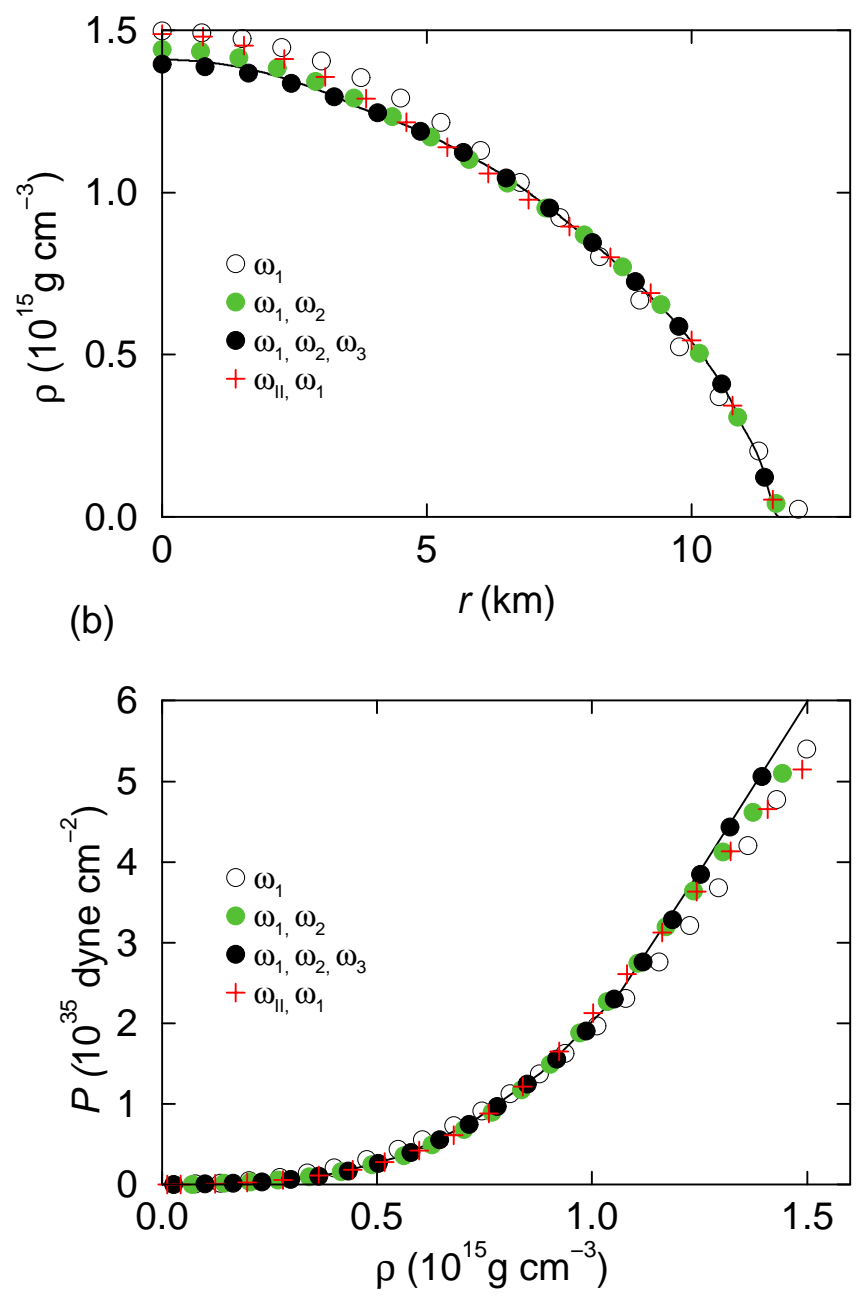

FIG. 5: Panels (a) and (b) depict $\rho(r)$ and $P(\rho)$ respectively for an APR1 star with $\mathcal{C}=0.28$. The solid line is the theoretical value, while the unfilled/grey/dark circles represent the results obtained from inversion scheme using one/two/three leading axial $w$-modes. The result obtained from inversion scheme using $\omega_{1}$ and the frequency of a $w_{\mathrm{II}}$-mode is shown by the crosses. 
(a)

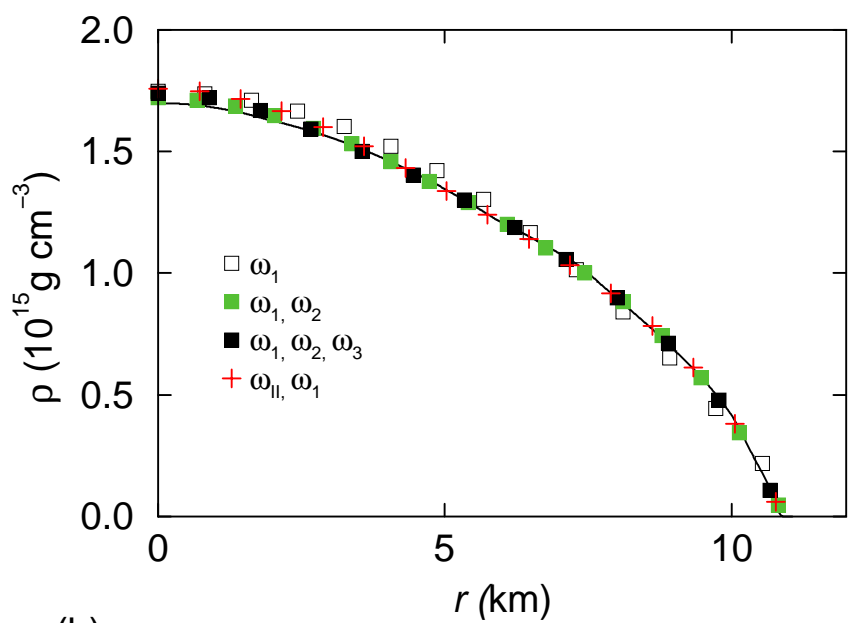

(b)

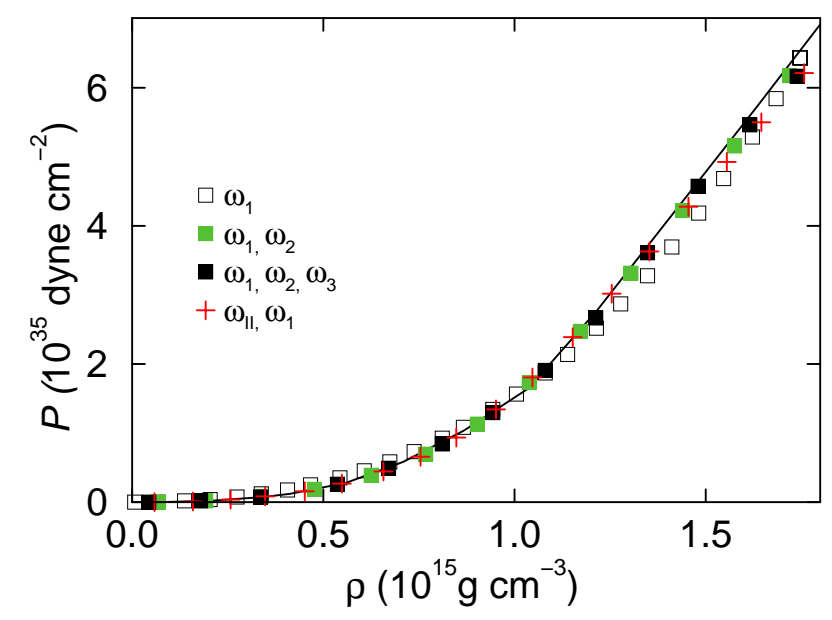

FIG. 6: Same as Fig. 5 except that an APR2 star is considered instead. 
(a)
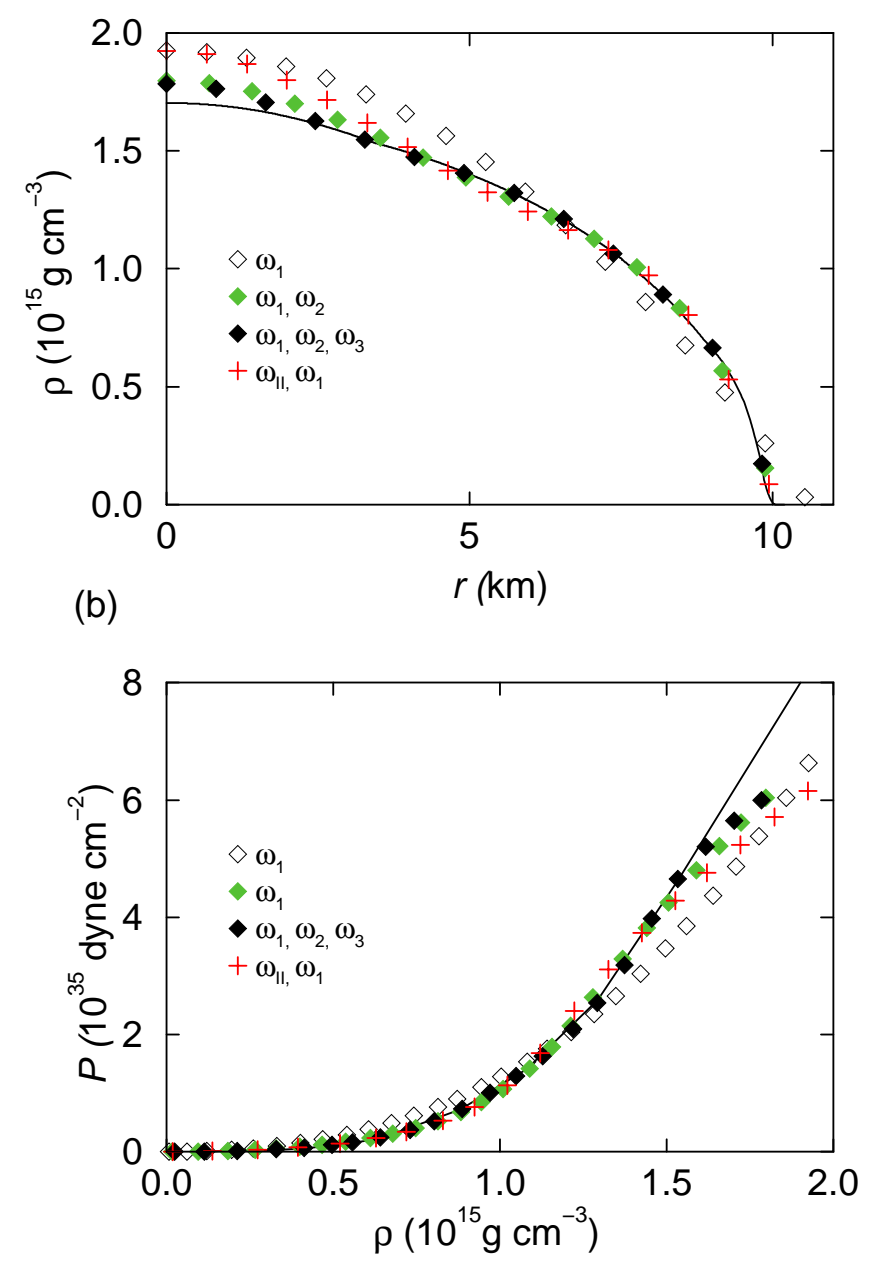

FIG. 7: Same as Fig. [5 except that an AU star is considered instead. 
(a)
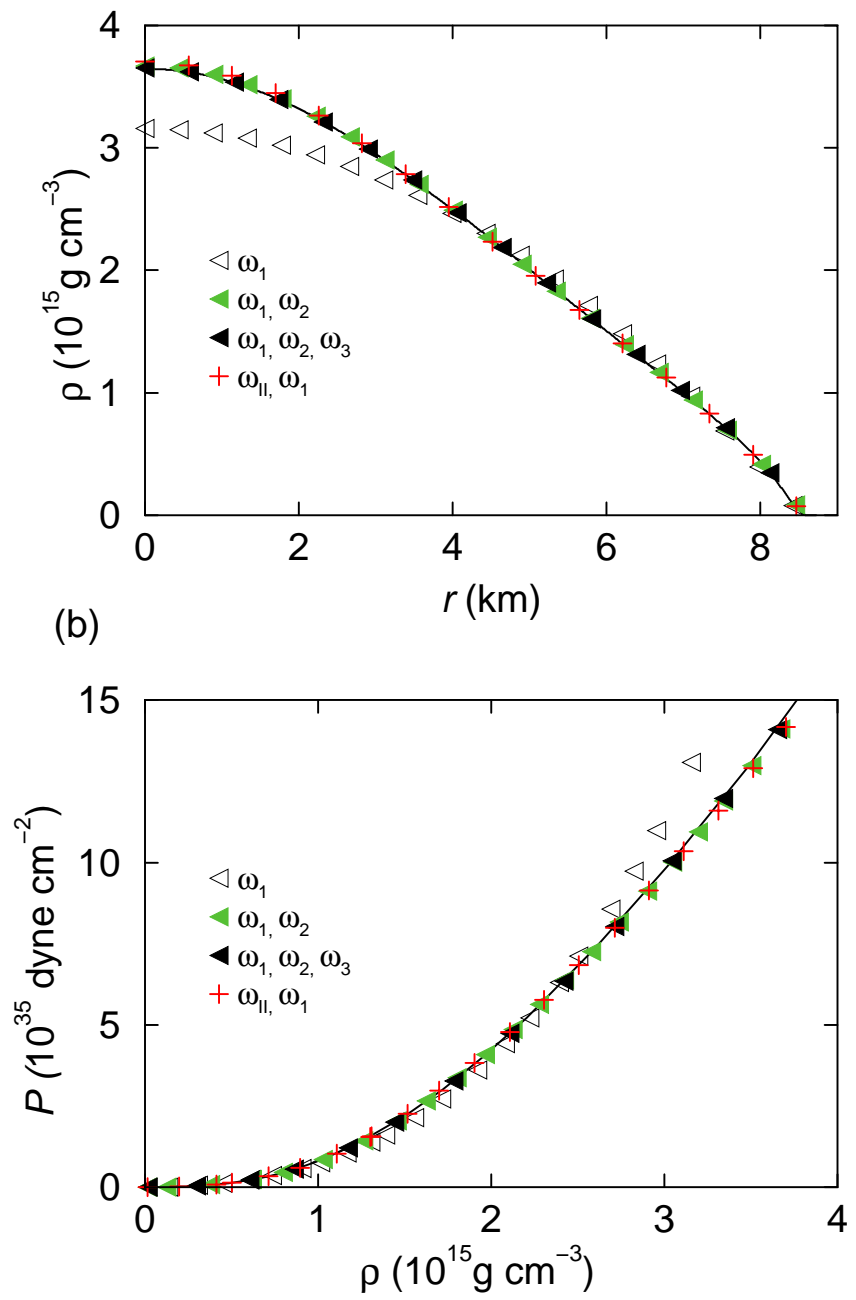

FIG. 8: Same as Fig. 5 except that a model A star is considered instead. 
(a)

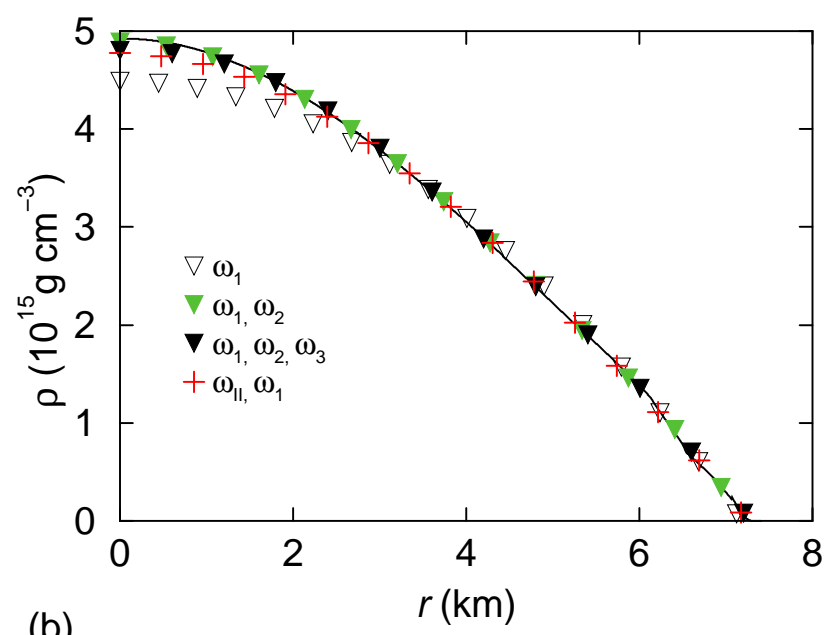

(b)

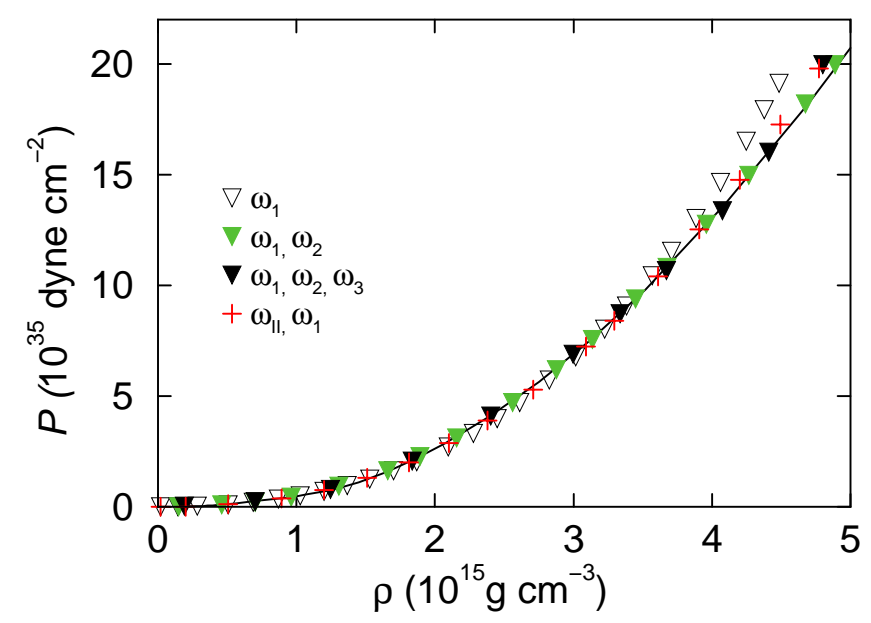

FIG. 9: Same as Fig. 5 except that a model C star is considered instead. 
(a)

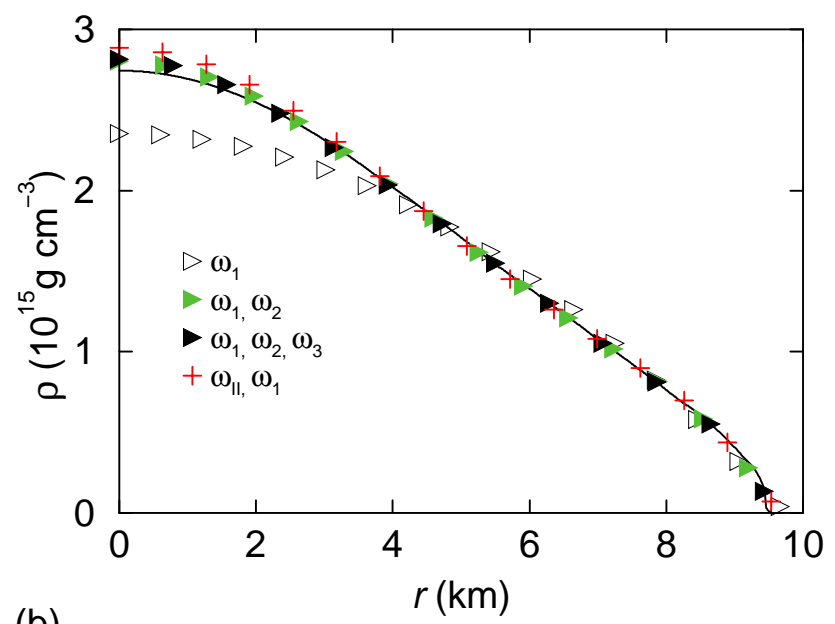

(b)

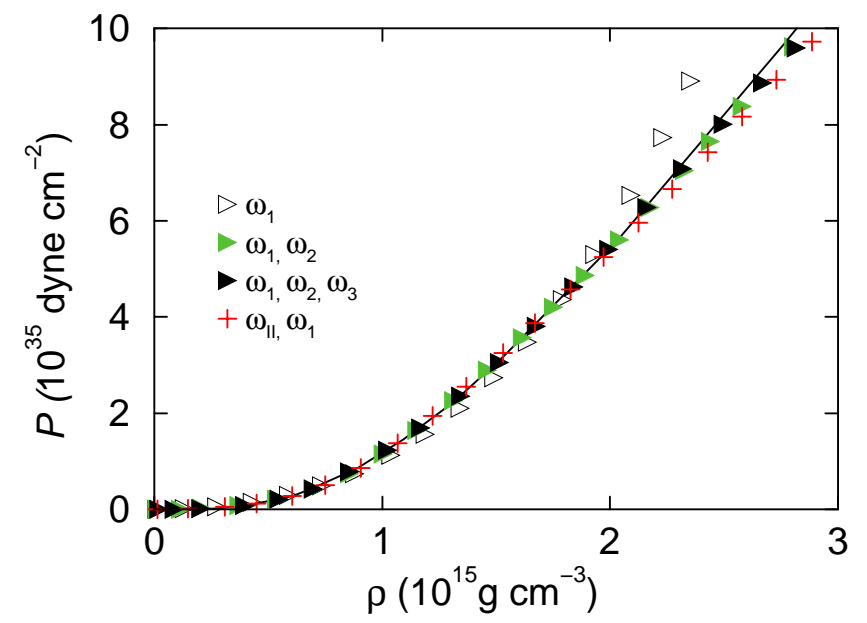

FIG. 10: Same as Fig. 5] except that a UT star is considered instead. 
(a)

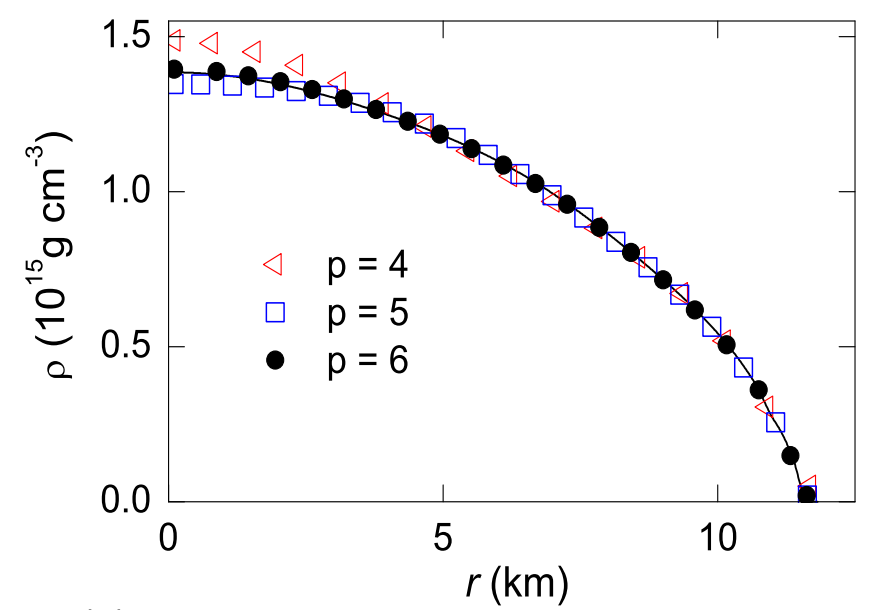

(b)

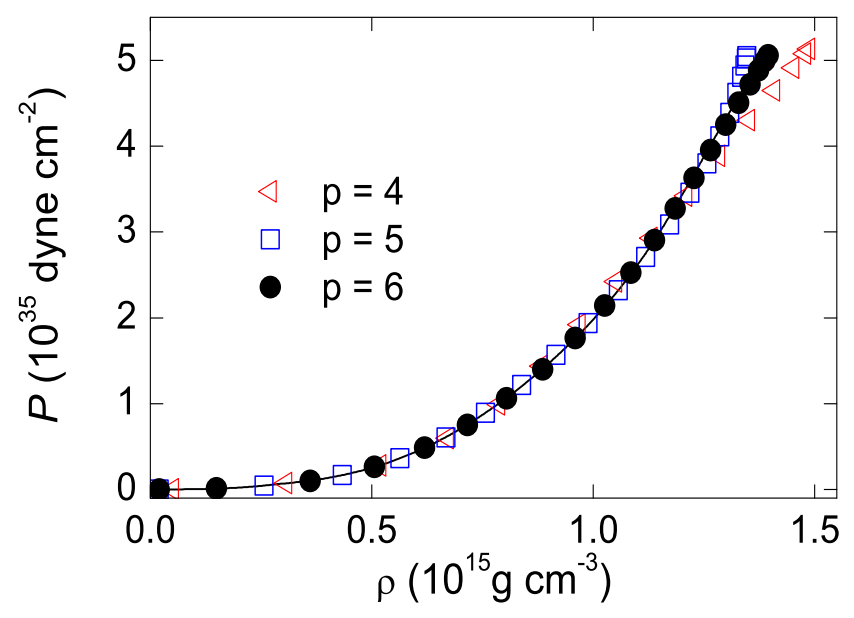

FIG. 11: Panels (a) and (b) depict $\rho(r)$ and $P(\rho)$ respectively for an APR1 star with $\mathcal{C}=0.28$. The solid line is the theoretical value, while the left triangles/squares/dark circles represent the results obtained from inversion scheme using four/five/six parameters and three leading axial $w$-modes. 

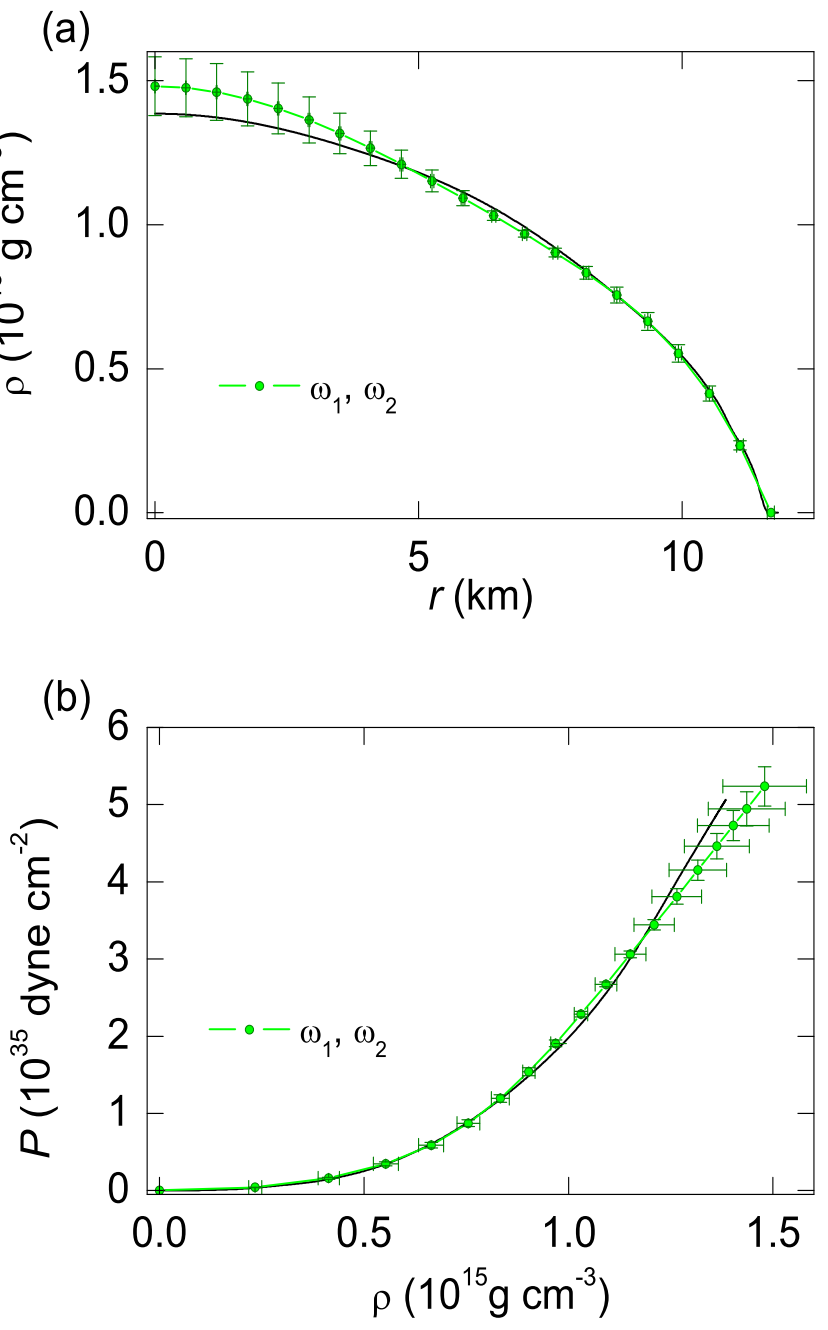

FIG. 12: Panels (a) and (b) depict $\rho(r)$ and $P(\rho)$ respectively for an APR1 star with $\mathcal{C}=0.28$. The solid line is the theoretical value, while the grey circles connected by a grey line represent the results obtained from inversion scheme using two approximately known leading axial $w$-modes and four parameters. Twelve sets of data were used in the simulation. The errors in the real and imaginary parts of the QNM frequency were uniformly distributed in a $\pm 5 \%$ interval. 
(a)

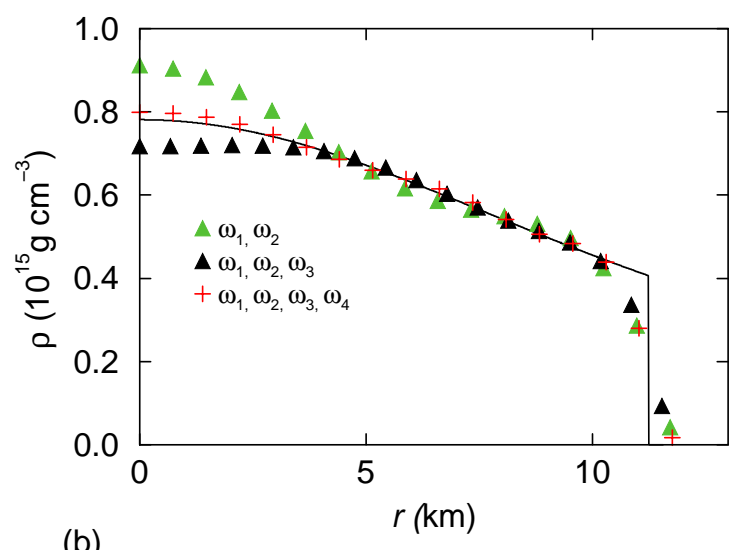

(b)

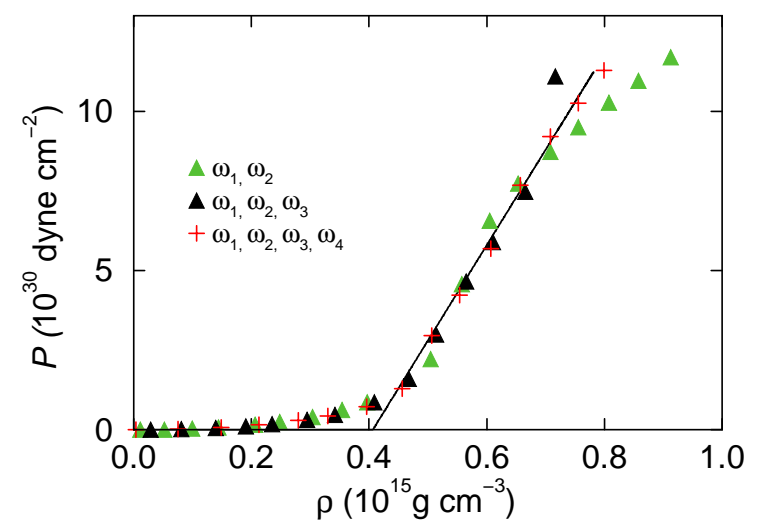

FIG. 13: Panels (a) and (b) depict $\rho(r)$ and $P(\rho)$ respectively for a SQS star with $\mathcal{C}=0.2$. The solid line is the theoretical value, while the grey triangles/dark triangles/crosses respectively represent the results obtained from inversion scheme using two, three and four leading axial $w$-modes.

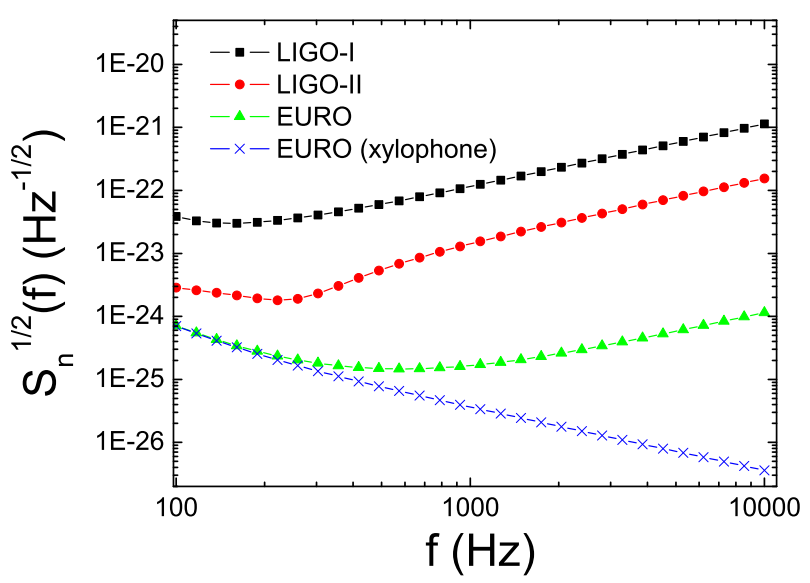

FIG. 14: The noise amplitude $S_{n}^{1 / 2}(f)\left(\mathrm{Hz}^{-1 / 2}\right)$ is plotted against $f(\mathrm{~Hz})$ for gravitational-wave detectors LIGO-I, LIGOII, EURO and EURO (xylophone). The curves are based on the interpolation formulas available at the EURO homepage http://www.astro.cf.ac.uk/geo/euro 